\title{
Phenomenology of diffractive DIS in the framework of fracture functions and determination of diffractive parton distribution functions
}

\author{
Hamzeh Khanpour* \\ Department of Physics, University of Science and Technology of Mazandaran, \\ P.O. Box 48518-78195 Behshahr, Iran \\ and School of Particles and Accelerators, Institute for Research in Fundamental Sciences (IPM), \\ P.O. Box 19395-5531 Tehran, Iran
}

(Received 11 February 2019; published 13 March 2019)

\begin{abstract}
The goal of this study is to determine a set of diffractive parton distribution functions (diffractive PDFs) from a QCD analysis of all available and up-to-date diffractive deep inelastic scattering (diffractive DIS) datasets from HERA $e p$ collider, including the most recent $\mathrm{H} 1$ and ZUES combined inclusive diffractive cross section measurements. This extraction of diffractive PDFs, referred to as HK19-DPDF, is performed at nextto-leading (NLO) and next-to-next-to-leading (NNLO) in perturbative quantum chromodynamics (QCD). This new determination of diffractive PDFs is based on the fracture functions methodology, a QCD framework designed to provide a statistically sound representation of diffractive DIS processes. Heavy quark contributions to the diffractive DIS are considered within the framework of the FONLL general mass variable flavor number scheme (GM-VFNS) and the "Hessian approach" is used to determine the uncertainties of diffractive PDFs. We discuss the novel aspects of the approach used in the present analysis, namely an optimized and flexible parametrization of the diffractive PDFs as well as a strategy based on the fully factorization theorem for diffractive hard processes. We then present the diffractive PDFs, and discuss the fit quality and the stability upon variations of the kinematic cuts and the fitted datasets. We find that the systematic inclusion of higher-order QCD corrections could improves the description of the data. We compare the extracted sets of diffractive PDFs based on the fracture functions approach to other recent sets of diffractive PDFs, finding in general very good agreements.
\end{abstract}

DOI: 10.1103/PhysRevD.99.054007

\section{INTRODUCTION}

In the past decades, there has been an increasing interest in attempting to understand the structure of the hadron $[1,2]$. The high energy processes of interest which contains information on the hadron structure in quantum chromodynamics (QCD) mostly include the hadron production in lepton-nucleon $(\ell p)$ deep-inelastic scattering (DIS) and in proton-proton $(p p)$ collisions. Information from hadron collisions, especially at the large hadron collider (LHC) at CERN, is particularly useful in order to achieve a precise understanding of nonperturbative QCD dynamics. Since its start of data taking, the $\mathrm{H} 1$ and ZEUS experiments at HERA-I and HERA-II have provided an impressive wealth of information on the quark and gluon structure of the proton, and hence, a considerable amount of literature has

\footnotetext{
*Hamzeh.Khanpour@mail.ipm.ir
}

Published by the American Physical Society under the terms of the Creative Commons Attribution 4.0 International license. Further distribution of this work must maintain attribution to the author(s) and the published article's title, journal citation, and DOI. Funded by SCOAP. been published over the past decade. Indeed, modern global analyses of parton distribution functions (PDFs) [3-6] as well as diffractive PDFs include a wide range of HERA measurements of electron-proton process. Recent developments in the field of PDFs of the proton have led to a renewed interest in the diffractive DIS process to extract the nonperturbative diffractive PDFs from a global analysis. Significant progress in understanding diffraction processes has been made at the HERA collider, where typically a $27.5 \mathrm{GeV}$ electron (or positron) collides with a 820 or $920 \mathrm{GeV}$ proton. Recent analyses of diffractive DIS data collected by $\mathrm{H} 1$ and ZEUS collaborations at HERA have confirmed substantial contributions of perturbative QCDbased effects in diffractive DIS cross sections. In general, in $e p$ collisions at HERA, the hard diffraction contributes a fraction of order $8 \%-10 \%$ to the total DIS cross sections.

Future high precision and high energy DIS experiments are expected to reach a wider kinematic range of momentum fraction $x$ and photon virtuality $\mathrm{Q}^{2}$ which could not be explored previously by ep HERA. Among them are the Large Hadron-electron Collider (LHeC) [7] and Future Circular Collider in electron-hadron mode (FCC-eh) $[8,9]$. LHeC would utilize the $7 \mathrm{TeV}$ proton beam from the LHC and 
collides it with a $60 \mathrm{GeV}$ electron beam, and would extend the available kinematic range in $x$ and photon virtuality $\mathrm{Q}^{2}$ by a factor of order 20 and 100, respectively. Beyond the $\mathrm{LHeC}$, the next generation ep collider FCC-eh, utilizing the $50 \mathrm{TeV}$ proton beam from the $\mathrm{FCC}$ which would probe DIS processes at center-of-mass energy of $3.5 \mathrm{TeV}$, much higher than the HERA collider, leading to a better understanding of the proton structure with extremely high precision. Recently, an investigation of the potential of theses high energy and high luminosity machines for the measurement of diffractive DIS cross sections and to constrain the diffractive PDFs has been performed, see the analysis of Ref. [10] for details.

On the phenomenological side, a similar strategy to determine the PDFs can also be adapted to the case of diffractive DIS, considering the collinear factorization [11] and the validity of proton vertex factorization [12]. Diffractive PDFs can be extracted from QCD analyses of diffractive DIS datasets. Similarly to the PDFs, the diffractive PDFs are expected to obey the Dokshitzer-Gribov-LipatovAltarelli-Parisi (DGLAP) equations. In this picture of proton vertex factorization, the diffractive DIS processes are described by the exchange of colorless object such a pomeron. Recent progress in the determination of diffractive PDFs widely used the diffractive DIS datasets from H1 and ZEUS experiments at HERA (see, for example, Refs. $[10,13,14]$ for recent reviews). In the last few years, at least three groups have reported sets of diffractive PDFs with uncertainties using the mentioned datasets: $\mathrm{H1}-2006$ DPDF [15], ZEUS-2010-DPDF [16], and the most recent analysis by GKG1 8 -DPDF [17]. All these of diffractive PDFs determinations were performed at next-to-leading order (NLO) accuracy in perturbative QCD. The primary focus of these QCD analyses were put on quantifying the effects of the inclusion of new measurements of diffractive DIS at HERA as well as the diffractive dijet production. The analysis by GKG18-DPDF introduced some improvements over previous determinations. Specifically, in order to achieve a more reliable estimate of the diffractive PDFs uncertainties, the XFITTER package have been employed. In addition, GKG18-DPDF used for the first time the most recent H1/ZEUS combined diffractive DIS cross section measurements. Up to now, predictions for diffractive DIS, and in particular for diffractive dijet production, were performed only at next-to-leading order (NLO) accuracy. Recently, predictions for the diffractive dijet production, also is provided at next-to-next-to-leading order (NNLO) in Ref. [18]. Although much theoretical work remains to be done for this process, it is already clear that the accumulation of precise data for diffractive DIS processes will greatly deepen our understanding of perturbative QCD (pQCD).

In the present study we construct for the first time a set of NLO and NNLO diffractive PDFs using all available and upto-date diffractive DIS cross section measurements [19,20] including the most recent $\mathrm{H} 1$ and ZEUS combined measurement for the inclusive diffractive DIS cross sections [21]. We do so by using a methodology which has been suggested in Refs. [22,23], and recently used to study leading neutron production [24-26] as well as inclusive diffractive DIS [27]: the so called "fracture function approach."

Similar to the case of ordinary structure functions in totally inclusive DIS, QCD does not predict the shape of the fracture functions unless it is known at a certain initial scale, $Q_{0}^{2}$. This universal and nonperturbative information has to be determined from a QCD analysis of experimental datasets, and hence, can be parametrized finding inspiration in nonperturbative models. More recently, the fracture functions approach has been successfully applied to describe leading neutron and leading proton productions at $\mathrm{H} 1$ and ZEUS experiment, considering a model as nonperturbative input [24-26].

The main aim of this analysis is to provide a conceptual theoretical framework based on the fracture function approach to describe the diffractive DIS processes. As a result of the following analysis, we present a parametrization for the fracture function that characterizes the underlying diffractive DIS process at an initial scale, as extracted from $\mathrm{H} 1$ and ZEUS datasets. The obtained parametrization is used to compute other observables measured by $\mathrm{H} 1$, not included in our QCD fit, finding also an outstanding agreement with the data. In this analysis we emphasize that an approach in the framework of fracture functions phenomenologically allows a accurate and reliable description of diffractive DIS cross sections. Our results also verify that the scale dependence of the diffractive DIS data agrees well with the one predicted by the use of fracture function formalism. Therefore, this paper provides an important opportunity to advance the understanding of diffractive DIS events measured by H1 and ZEUS collaboration at HERA.

This paper has been organized in the following way: Sec. II begins by laying out the theoretical framework and assumptions of this research. In particular, we discuss the methodology used for this study including the diffractive structure functions, diffractive PDFs, hard-scattering factorization, and heavy quark contributions. Then in Sec. III we present our input for the diffractive PDFs at a given initial scale $Q^{2}=Q_{0}^{2}$. In Sec. IV we concentrate on the description of the H1 datasets which have been added in our QCD analysis, together with a discussion on the inclusion of $\mathrm{H} 1$ and ZEUS combined datasets. Section V is concerned with the methodology used in this study to determine the diffractive PDFs uncertainty. The results of the global analysis can be found in Sec. VI. This section starts with a presentation of our NLO and NNLO diffractive PDFs and their uncertainties, together with the values of the input parameters. Detailed discussions of the main results and comparisons with the analyzed datasets are also presented as well. Finally, the conclusion in Sec. VII gives a brief summary and critique of the findings.

\section{THEORETICAL FRAMEWORK AND ASSUMPTIONS}

Predictions for the diffractive processes in DIS can be obtained in the framework of pQCD. As we already 
mentioned, hard processes in diffractive DIS can be described by a factorization into parton level subprocesses and diffractive PDFs. In this framework, cross sections for diffractive deep inelastic electron proton $(e p)$ scattering can be computed at NLO and NNLO QCD accuracy.

\section{A. Diffractive structure functions}

The kinematical variables to describe the diffractive DIS can be inferred from the momenta of the incoming lepton, proton and the outgoing lepton. The leading order Feynman diagram for diffractive DIS at HERA is displayed in Figs. 1 and 2 in the picture of proton vertex and collinear and QCD hard scattering collinear factorizations, respectively. In the diffractive DIS in which belongs to the semi-inclusive lepton proton DIS,

$$
\ell(k)+p(P) \rightarrow \ell\left(k^{\prime}\right)+p\left(P^{\prime}\right)+X\left(p_{X}\right),
$$

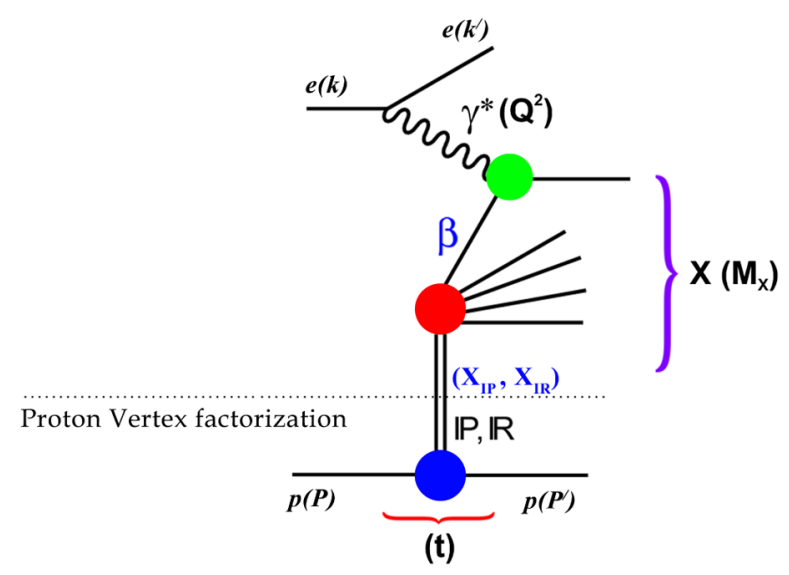

FIG. 1. Representative Feynman diagram for the neutral current diffractive DIS process $e p \rightarrow e p X$ proceeding via virtual photon exchange in the picture of proton vertex factorization. The kinematic variables are described in the text.

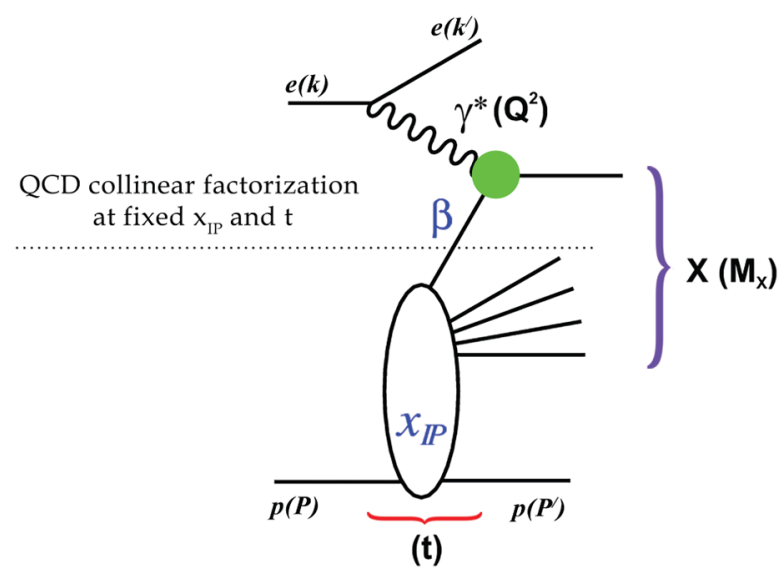

FIG. 2. Representative Feynman diagram for the neutral current diffractive DIS process $e p \rightarrow e p X$ proceeding via virtual photon exchange in the picture of QCD hard scattering collinear factorization. The kinematic variables are described in the text. in which along with the outgoing leptons, an additional proton $p$ can be detected in the final state. In the above equation, $X$ stands for the unobserved part of the hadronic final state. The kinematics of such events is specified by the following variables:

$$
Q^{2}=-q^{2}, \quad x_{B}=\frac{Q^{2}}{2 P \cdot q}, \quad y=\frac{P . q}{P . k} .
$$

The momentum transferred to the target proton is given by the momentum $q^{2}=\left(k-k^{\prime}\right)^{2}$ of the virtual photon $\gamma^{*}$. Hence, the $Q^{2}$ in Eq. (2) is the photon virtuality. $x_{B}$ is the usual $x$-Bjorken variable in DIS which is the longitudinal momentum fraction of the proton carried by the struck quark, and $y$ is refereed to as the inelasticity of the scattering.

In the lepton-proton center-of-mass system, diffractive DIS events are then characterized by outgoing protons with a large momentum fraction of the incident proton and quite small values of the transverse momentum measured with respect to the collisions axis, i.e., in the target fragmentation region of the incident protons.

In order to the describe such diffractive events, additional kinematics invariants have to be introduced. As illustrated in Fig. 2, the longitudinal momentum fractions, $x_{\mathbb{P}}$ of the colorless exchange with respect to the incoming proton momentum, and the invariant momentum transfer $t$ at the proton vertex $t$, and $\beta$ of the struck quark with respect to the colorless exchange, are needed to describe diffractive DIS. The two new variables are given by,

$$
x_{\mathbb{P}}=\frac{q \cdot\left(P-P^{\prime}\right)}{P \cdot q}, \quad \beta=\frac{Q^{2}}{Q^{2}+M_{X}^{2}} .
$$

$M_{X}$ is the invariant mass of the hadronic final state $X$. The scaled fractional momentum variable $\beta$ which is also defined by $\beta=\frac{x_{B}}{x_{\mathrm{P}}}$ interpreted as the fractional momentum of interacting parton in the proton with respect to the Pomeron fractional momentum $x_{\mathbb{P}}$.

Now we turn to inclusive diffractive DIS cross sections. The neutral current measurements in diffractive DIS are often presented in terms of the reduced lepton-proton $(\ell p)$ cross section, "diffractive reduced cross section" $\sigma_{r}^{D(4)}$. The $t$-unintegrated reduced cross section depends on the diffractive transverse and longitudinal structure functions $F_{2}^{D(4)}$ and $F_{L}^{D(4)}$, respectively. In the one-photon exchange approximation, it is given by:

$$
\begin{aligned}
\sigma_{r}^{D(4)}\left(\beta, Q^{2} ; x_{\mathbb{P}}, t\right)= & F_{2}^{D(4)}\left(\beta, Q^{2} ; x_{\mathbb{P}}, t\right) \\
& -\frac{y^{2}}{1+(1-y)^{2}} F_{L}^{D(4)}\left(\beta, Q^{2} ; x_{\mathbb{P}}, t\right) .
\end{aligned}
$$

In the measurements of inclusive diffractive DIS at HERA, the data were presented in terms of $\sigma_{r}^{D(4)}\left(\beta, Q^{2} ; x_{\mathbb{P}}\right)$. In this analysis, we analyze all available and up-to-date 
diffractive DIS datasets $[19,20]$ including the most recent data for combined $\mathrm{H} 1$ and ZEUS diffractive DIS cross sections measurements [21].

\section{B. Diffractive parton distributions and hard-scattering factorization}

According to the factorization theorem for diffractive DIS in pQCD [11,28,29], if the process is sufficiently hard, the calculation can be subdivided into two components: the hard partonic cross sections which are calculable within the pQCD in powers of strong coupling constant $\alpha_{s}$, which need to be convoluted with soft diffractive PDFs, $\mathcal{F}_{i / p}^{D}\left(\beta, \mu_{F}^{2} ; x_{\mathbb{P}}, t\right)$ that specify the contributing parton inside the incoming hadron. Likewise the PDFs, diffractive PDFs are also universal for all diffractive DIS with the hardness of the process being ensured by the virtuality $Q^{2}$ of the exchanged photon. The structure functions appearing in Eq. (4) are given by

$$
\begin{aligned}
F_{k}^{D(4)}\left(\beta, Q^{2} ; x_{\mathbb{P}}, t\right)= & \sum_{i} \int_{\beta}^{1} \frac{d \zeta}{\zeta} \mathcal{F}_{i / p}^{D}\left(\beta, \mu_{F}^{2} ; x_{\mathbb{P}}, t\right) \\
& \times C_{k i}\left(\frac{\beta}{\zeta}, \frac{Q^{2}}{\mu_{F}^{2}}, \alpha_{s}\left(\mu_{R}^{2}\right)\right)+\mathcal{O}\left(\frac{1}{Q^{2}}\right) .
\end{aligned}
$$

The index $i$ in Eq. (5) runs on the flavor of the interacting partons in the proton [27]. The hard scattering coefficient functions, $C_{q}$ and $C_{g}$, which appeared in above equation are perturbatively calculable as a power expansion in the strong coupling and are the same as in fully inclusive DIS process [30]. As one can find in literature, for example [15-17, 19-21,31-33] as well as our discussion in previous section, in order to describe the diffractive DIS events, two more variable in addition to the usual DIS kinemtical variables are also needed which are the longitudinal momentum fraction $x_{\mathbb{P}}$ and the invariant momentum transfer $t$. In the general factorization theorem $[11,28,29]$ for the diffractive DIS in the form of above equation holds at fixed values of $x_{\mathbb{P}}$ and invariant momentum transfer $t$ [27]. Hence, the parton content of the color singlet exchange described by diffractive PDFs, $\mathcal{F}_{i / p}^{D}$, is uniquely controlled by the kinematics of the outgoing proton with the fractional momentum of $1-x_{\mathbb{P}}$.

The diffractive PDFs, $\mathcal{F}_{i / p}^{D}\left(\beta, \mu_{F}^{2} ; x_{\mathbb{P}}, t\right)$, appearing in Eq. (5) are proton-to-proton fracture functions [22] in the very forward kinematical region. It can be interpreted as the number density of interacting partons at a scale $\mu^{2}$ with fractional momentum of $\beta$ conditional to the detection of a final state proton with fractional momentum $1-x_{\mathbb{P}}$ and invariant momentum transfer $t$. As in the inclusive case, the $Q^{2}$ evolution of the diffractive DIS can be predicted in pQCD. Since the scale dependence of the cross section in leading particle production in DIS can be calculated within pQCD [22,34], therefore the diffractive PDFs also obey the standard DGLAP evolution equations [24-26,34,35]. The $t$-unintegrated diffractive PDFs appearing in Eq. (5) also obey the standard DGLAP evolution equations [24,27].

By integrating Eq. (5) with respect to the momentum transfer $t$ over up to values of order $\mathrm{Q}^{2}$, the diffractive PDFs obey an inhomogeneous DGLAP-type evolution equations [22]. We should note here that most diffractive DIS events occur for the small values of $t$ and for the case of H1/ZEUS combined data, the squared four-momentum transfer at the proton vertex, $t$, is integrated in the restricted range of $0.09<|t|<0.55 \mathrm{GeV}^{2}$. In the case where the diffractive PDFs presented in Eq. (5) are integrated over $t$ in a limited range, they obey the standard DGLAP evolution equation [27,34]. Hence, we considered such approximation in our analysis and therefore one can use the standard DGLAP evolution when $\mathcal{F}_{i / p}^{D}\left(\beta, \mu_{F}^{2} ; x_{\mathbb{P}}, t\right)$ are integrated over $t$ in a limited range $[27,34]$

$$
\begin{aligned}
\mathcal{F}_{i / p}^{D}\left(\beta, \mu_{F}^{2} ; x_{\mathbb{P}}\right) & =\int_{t_{\min }}^{t_{\max }} d t \mathcal{F}_{i / p}^{D}\left(\beta, \mu_{F}^{2} ; x_{\mathbb{P}}, t\right), \\
t_{\max } & \ll Q^{2} .
\end{aligned}
$$

Hence, like for the case of PDFs, the evolution equations of diffractive PDFs are easily obtained by the DGLAP evolution equations $[22,34]$ as

$$
\begin{aligned}
Q^{2} & \frac{\partial \mathcal{F}_{\Sigma / p}^{D}\left(\beta, Q^{2} ; x_{\mathbb{P}}\right)}{\partial Q^{2}} \\
& =\frac{\alpha_{s}\left(Q^{2}\right)}{2 \pi} \int_{\beta}^{1} \frac{d u}{u} P_{\Sigma}^{j}(u) \mathcal{F}_{\Sigma / p}^{D}\left(\frac{\beta}{u}, Q^{2} ; x_{\mathbb{P}}\right), \\
Q^{2} & \frac{\partial \mathcal{F}_{g / p}^{B}\left(\beta, Q^{2} ; x_{\mathbb{P}}\right)}{\partial Q^{2}} \\
& =\frac{\alpha_{s}\left(Q^{2}\right)}{2 \pi} \int_{\beta}^{1} \frac{d u}{u} P_{g}^{j}(u) \mathcal{F}_{g / p}^{D}\left(\frac{\beta}{u}, Q^{2} ; x_{\mathbb{P}}\right),
\end{aligned}
$$

where $\mathcal{F}_{\Sigma / p}^{D}\left(\beta, Q^{2} ; x_{\mathbb{P}}\right)$ and $\mathcal{F}_{g / p}^{B}\left(\beta, Q^{2} ; x_{\mathbb{P}}\right)$ correspond to the singlet and gluon diffractive distributions, respectively. These nonperturbative distributions need to be parametrized at an input scale $Q_{0}^{2}$ and their evolution to higher scale, $Q^{2}>Q_{0}^{2}$, can be described by using the evolution equation given above. $P_{\Sigma}$ and $P_{g}$ in Eq. (7) are the common NLO and NNLO contributions to the splitting functions governing the evolution of unpolarized singlet and nonsinglet combinations of quark densities in perturbative QCD. Splitting functions are perturbatively calculable as a power expansion in the strong coupling constant $\alpha_{s}$. The splitting functions $P_{\Sigma}$ and $P_{g}$ in Eq. (7) are the same as in fully inclusive DIS [36,37].

We have now, in principle, all the essential ingredients to write down the diffractive DIS cross sections of Eq. (4) in terms of diffractive structure functions. In the next sections, 
we give a detailed account of the global analysis of diffractive PDFs performed in this study. We first discuss the heavy flavour contributions, the details parametrization of diffractive PDFs and then we will present data selection and the determination of the best fit, which we compare to the fitted data. We then focus on the studies of uncertainties using the standard "Hessian" error matrix approach.

\section{Heavy flavor contributions to the diffractive DIS structure function}

It is shown in literature that the correct treatments of heavy flavors have an important impact on the PDFs extracted from the global analysis due to the data available for heavy flavor structure functions, $F_{L}^{h}\left(x, Q^{2}, m_{h}^{2}\right)$ with $h=c, b$ and $k=2, L$, as well as the heavy flavor contributions to the total inclusive structure functions at small values of momentum fraction $x[4,38-40]$. There are several scheme available in literature where heavy quark production can be readily described. Among them are zeromass variable flavor scheme (ZM-VFNS), fixed-flavor number scheme (FFNS) and general-mass variable flavour number scheme (GM-VFNS).

The resulting calculations for the heavy quark coefficient functions are more accurate in two different and complementary regimes. The FFNS is more accurate for values of $\mathrm{Q}^{2}$ comparable to the mass of the heavy quark involved in the calculation, while the ZM-VFNS is instead more accurate for values of $\mathrm{Q}^{2}$ much larger than the heavyquark mass, $Q^{2} \gg m_{h}^{2}$. It has been shown in literature that the GM-VFNS is the most appropriate scheme to extract PDFs from a global QCD analysis [41-43]. This scheme could extrapolates smoothly from the FFNS at low value of $\mathrm{Q}^{2}$ to the ZM-VFNS at high $\mathrm{Q}^{2}$, and therefore, produces a good description of the effect of heavy quark contributions to the structure functions as well as inclusive cross section over the whole range of $\mathrm{Q}^{2}$. Well known examples of GM-VFNS are the original Aivazis-Collins-Olness-Tung (ACOT GM-VFNS) scheme [44], the Thorne-Roberts (TR GM-VFNS) scheme [45] and the so-called FONLL GM-VNFS scheme [46]. In our previous diffractive PDFs analysis, GKG18-DPDF [17] we adopted the more recent "TR" prescription [47].

In the present analysis, we prefer to use the FONLL GMVFNS [46] which provides the charm structure functions by using the exact $x$-space $\mathcal{O}\left(\alpha_{s}^{2}\right)$ heavy quark coefficient functions as well as computes neutral- and charged-current DIS observables up to this order. The evolution of diffractive PDFs $\mathcal{F}^{D}\left(\beta, Q^{2} ; x_{\mathbb{P}}\right)$ is performed within the FONLL GM-VFNS by using the publicly available APFEL program [48]. The QCD parameters are the ones quoted in the analysis by NNPDF Collaboration [49,50]. In particular, we use the heavy flavor masses for charm and bottom as $m_{c}=1.51 \mathrm{GeV}$ and $m_{b}=4.92 \mathrm{GeV}$, respectively. Also the $Z$-boson mass is chosen to be $M_{Z}=$ $91.1876 \mathrm{GeV}$ and the strong coupling is evaluated at two loop setting $\alpha_{\mathrm{s}, \mathrm{NLO}}^{n_{F}=5}\left(M_{Z}\right)=0.1185$ [51]. This selection of $\alpha_{s}\left(M_{Z}\right)$ is consistent with the very recent determination of the strong coupling constant reported by the NNPDF 3.1 [52], in which for the first time uses the jet production and $t \bar{t}$ differential distributions at NNLO accuracy.

\section{INPUT DISTRIBUTIONS}

In this section, we present the input distributions for the diffractive PDFs at the reference scale of $Q^{2}=Q_{0}^{2}$. We also glance ahead to mention some of the main feature of this selection. As is clear from the discussion in Sec. II B, one improvement in the following analysis is to use parametrizations for the input diffractive PDFs based on the fracture function approach. Following the detailed studies in Refs. $[25,26]$, we take for most PDFs a parametrization of the form

$$
\begin{aligned}
& \beta \mathcal{F}_{q}\left(\beta, Q_{0}^{2} ; x_{\mathbb{P}}\right)=\mathcal{W}\left(x_{\mathbb{P}}\right) \beta f_{q}\left(\beta, Q_{0}^{2}\right), \\
& \beta \mathcal{F}_{g}\left(\beta, Q_{0}^{2} ; x_{\mathbb{P}}\right)=\mathcal{W}\left(x_{\mathbb{P}}\right) \beta f_{g}\left(\beta, Q_{0}^{2}\right),
\end{aligned}
$$

where $Q_{0}^{2}=2 \mathrm{GeV}^{2}$ is the input scale, and $\mathcal{W}\left(x_{\mathbb{P}}\right)$ is the flux factor which we assume that it depends only on the $x_{\mathbb{P}}$ variable. For the diffractive flux factor, we use the standard functional form which can be written as,

$$
\mathcal{W}\left(x_{\mathbb{P}}\right)=x_{\mathbb{P}}^{w_{1}}\left(1-x_{\mathbb{P}}\right)^{w_{2}}\left(1+w_{3} x_{\mathbb{P}}^{w_{4}}\right) .
$$

The global fit determines the values of the set of parameters $w_{1}, w_{2}, w_{3}$, and $w_{4}$ for diffractive quark and gluon PDFs. We consider that this flux factor to be the same for the light sea and gluon, as there is not enough data which can constrain these distributions, while leaving all four parameters $w_{i}$ in the polynomial free leads to instabilities in the fit. The dependence of the diffractive PDFs on the $x_{\mathbb{P}}$ depends very much on the parametrization that one chooses for the flux factor. In Eq. (8), $\beta f_{q}\left(\beta, Q_{0}^{2}\right)$ and $\beta f_{g}\left(\beta, Q_{0}^{2}\right)$ are the quark and gluon densities at the reference scale $Q_{0}^{2}$. The quark and gluon densities depend on the scaled fractional momentum variable $\beta$ and photon virtuality $Q^{2}$.

$\beta f_{q}\left(\beta, Q_{0}^{2}\right)=\mathcal{N}_{q} \beta^{\alpha_{q}}(1-\beta)^{\beta_{q}}\left(1+\gamma_{q} \sqrt{\beta}+\eta_{q} \beta^{2}\right)$,
$\beta f_{g}\left(\beta, Q_{0}^{2}\right)=\mathcal{N}_{g} \beta^{\alpha_{g}}(1-\beta)^{\beta_{g}}\left(1+\gamma_{g} \sqrt{\beta}+\eta_{g} \beta^{2}\right)$.

For the quark density $\beta f_{q}\left(\beta, Q_{0}^{2}\right)$, we consider a standard functional parametrization. Not all the parameters in our inputs for the quark density are free. We will return to this issue that due to the lack of enough diffractive DIS datasets, one needs to fixed some of these variables at their best fit values, and therefore, there are potentially less free parameters in the diffractive PDFs fit rather than what we 
presented in Eq. (10). Like the case of quark density, the poorly determined gluon density $\beta f_{g}\left(\beta, Q_{0}^{2}\right)$ is also taken to has a simpler input functional form as presented in Eq. (10). It is worthwhile to mention here that our parametrizations for the input distributions and all free parameters listed there allow the QCD fits a large degree of flexibility.

Considering the flux factor introduced in Eq. (9) as well as the quark and gluon densities presented in Eq. (10), one can express the diffractive PDFs in terms of the following set of basis functions for the quark and gluon diffractive PDFs,

$$
\begin{aligned}
& \beta \mathcal{F}_{q}\left(\beta, Q_{0}^{2} ; x_{\mathbb{P}}\right)=\mathcal{W}\left(x_{\mathbb{P}}\right) \mathcal{N}_{q} \beta^{\alpha_{q}}(1-\beta)^{\beta_{q}}\left(1+\gamma_{q} \sqrt{\beta}+\eta_{q} \beta^{2}\right), \\
& \beta \mathcal{F}_{g}\left(\beta, Q_{0}^{2} ; x_{\mathbb{P}}\right)=\mathcal{W}\left(x_{\mathbb{P}}\right) \mathcal{N}_{g} \beta^{\alpha_{g}}(1-\beta)^{\beta_{g}}\left(1+\gamma_{g} \sqrt{\beta}+\eta_{g} \beta^{2}\right) .
\end{aligned}
$$

As already emphasized, the introduction of such flexible parameterization for the diffractive PDFs at the reference scale gives a much better description of the diffractive DIS data. Equation (11) states that the variables $x_{\mathbb{P}}$, which related to the loosely scattered proton in diffractive DIS, are factorized from the variables characterizing the diffractive system $\left(\beta, Q^{2}\right)$. As we mentioned in Sec. II B, for a fixed value of $x_{\mathbb{P}}$, the $\mathrm{Q}^{2}$ evolution of diffractive PDFs $\beta \mathcal{F}\left(\beta, Q^{2} ; x_{\mathbb{P}}\right)$ is given by the DGLAP equations.

\section{DIFFRACTIVE DIS DATASETS}

We strive to include as much of the available diffractive DIS data as possible in our datasets. However, one needs to apply some certain kinematical cuts in order to ensure that only the datasets for which the available perturbative QCD treatment is adequate are included in the QCD fit. All the datasets used in the GKG18 diffractive PDFs analysis are also included in this analysis. Statistically, most significant dataset that we use in our QCD analysis are the HERA measurements of the diffractive DIS "reduced" cross sections. An overview of all available and most up-to-date diffractive DIS dataset is presented in Table I, where we indicate, for each dataset: the name of experiments and the corresponding references, observables, the kinematic range of $\beta, x_{\mathbb{P}}$ and $Q^{2}$, and finally the number of data points before kinematic cuts.

We now discuss the inclusion of the HERA diffractive DIS datasets into our diffractive PDFs fit. The datasets that are included in our analysis are as follow: The $\mathrm{H} 1$ and ZEUS combined diffractive DIS cross section measurement [21], the H1-LRG-12 [20] data and finally the H1-LRG-11 [19] data for three different center-of-mass energies of $\sqrt{s}=225,252$ and $319 \mathrm{GeV}$.

Now we are in a position to present the details of these datasets in turn. As we mentioned, in our QCD fit, we use the most recent $\mathrm{H} 1$ and ZEUS combined measurement for inclusive diffractive scattering cross sections [21]. Until recently, most of the other groups in literature that have performed global diffractive PDFs analyses do not include this combined datasets. An exception is the analysis of Ref. [17] by GKG18-DPDF. In the present work, and in GKG1 8 -DPDF, we have added the H1/ZEUS combined data in order to present a well-determined diffractive PDFs with a reliable uncertainty. This combined dataset contains complete information on diffractive DIS cross sections measurement published by the $\mathrm{H} 1$ and ZEUS Collaborations. The kinematic range of these combined datasets is $2.5 \mathrm{GeV}^{2}<Q^{2}<200 \mathrm{GeV}^{2}$ in photon virtuality, $3.5 \times 10^{-4}<x_{\mathbb{P}}<9.0 \times 10^{-2}$ in proton fractional momentum loss, $1.8 \times 10^{-3}<\beta<0.816$ in scaled fractional momentum variable and finally $0.09<|t|<$ $0.55 \mathrm{GeV}^{2}$ in squared four momentum transfer at the proton vertex. This measurement used samples of diffractive DIS data at a center-of-mass energy of $\sqrt{s}=318 \mathrm{GeV}$. The combination by $\mathrm{H} 1$ and ZEUS Collaborations is based on the cross sections measured with the ZEUS LPS 1 [53], ZEUS LPS 2 [54], H1 FPS HERA I [55] and finally the H1 FPS HERA II [56] datasets.

Another dataset we have used in our analysis is the recent inclusive measurement of diffractive DIS at HERA by $\mathrm{H} 1$ Collaboration, entitled as H1-LRG-12 [20]. The

TABLE I. List of all available and most up-to-date diffractive DIS data points (before applying the kinematical cuts) used in HK19DPDF global analysis. For each dataset we have provided the experiment and the corresponding reference, the kinematical coverage of $\beta$, $x_{\mathbb{P}}$, and $Q^{2}$ and the number of data points. The details of each experiment explained in the text.

\begin{tabular}{lccccccc}
\hline \hline Experiment & Observable & {$\left[\beta_{\min }, \beta_{\max }\right]$} & {$\left[x_{\mathbb{m}}^{\min }, x_{\mathbb{P}}^{\max }\right]$} & $Q^{2}\left[\mathrm{GeV}^{2}\right]$ & \# of points & $\mathcal{N}^{\text {NLO }}$ & $\mathcal{N}^{\text {NNLO }}$ \\
\hline H1/ZEUS Combined [21] & $\sigma_{r}^{D(3)}$ & {$[0.0018,0.562]$} & {$\left[3.0 \times 10^{-4}, 9.0 \times 10^{-2}\right]$} & $2.5-200$ & $\mathbf{1 8 1}$ & 0.9997 & 0.9996 \\
H1-LRG-12 [20] & $\sigma_{r}^{D(3)}$ & {$[0.0017,0.80]$} & {$\left[3.0 \times 10^{-4}, 3.0 \times 10^{-2}\right]$} & $3.5-1600$ & $\mathbf{2 6 7}$ & 0.9998 & 0.9997 \\
H1-LRG-11 $\sqrt{s}=225[19]$ & $\sigma_{r}^{D(3)}$ & {$[0.033,0.699]$} & {$\left[5.0 \times 10^{-4}, 3.0 \times 10^{-3}\right]$} & $4.0-44$ & $\mathbf{2 0}$ & 1.0036 & 1.0049 \\
H1-LRG-11 $\sqrt{s}=252[19]$ & $\sigma_{r}^{D(3)}$ & {$[0.089,0.699]$} & {$\left[5.0 \times 10^{-4}, 3.0 \times 10^{-3}\right]$} & $4.0-44$ & $\mathbf{1 9}$ & 1.0036 & 1.0049 \\
H1-LRG-11 $\sqrt{s}=319[19]$ & $\sigma_{r}^{D(3)}$ & {$[0.089,0.699]$} & {$\left[5.0 \times 10^{-4}, 3.0 \times 10^{-3}\right]$} & $11.5-44$ & $\mathbf{1 2}$ & 1.0036 & 1.0049 \\
Total data & & & & & & & \\
\hline \hline
\end{tabular}


measurement is restricted to the phase space region $3<$ $Q 2<1600 \mathrm{GeV}^{2}$ of the photon virtuality, the square of the four momentum transfer at the proton vertex with $|t|<$ $1.0 \mathrm{GeV}^{2}$ and finally the longitudinal momentum fraction of the incident proton $x_{\mathbb{P}}<0.05$ carried by the colorless exchange. These high statistics measurements at HERA cover the data taking periods of 1999-2000 and 2004-2007. This measurement is combined with previously published results by $\mathrm{H} 1$ Collaboration [15] in order to provide a single dataset of diffractive DIS cross sections using the large rapidity gap (LRG) selection method. Like for the case of H1/ZEUS combined datasets, this measurement is also presented as the reduced diffractive cross section, $\sigma_{r}^{D(4)}\left(\beta, Q^{2} ; x_{\mathbb{P}}, t\right)$.

Finally, we have used $\mathrm{H} 1$ measurements of the diffractive DIS reduced cross section [19] at center-of-mass energies of $\sqrt{s}=225,252 \mathrm{GeV}$ as well as the precise measurement at $\sqrt{s}=319 \mathrm{GeV}$. This reduced cross section measurements in this experiment, entitled H1-LRG-11, is measured in the photon virtuality range of $4.0<Q^{2}<44.0 \mathrm{GeV}^{2}$ and longitudinal momentum fraction of the diffractive exchange $x_{\mathbb{P}}$ of $5 \times 10^{-4}<x_{\mathbb{P}}<3 \times 10^{-3}$.

To ensure the validity of the well-known DGLAP evolution equations one needs to impose certain cuts on the datasets we discussed in this section. We follow the analysis by H1-2006 DPFs [15] and continue to use the same cuts on the diffractive DIS data, i.e., $Q^{2}>Q_{\min }^{2}=$ $8.5 \mathrm{GeV}^{2}$ in order to make sure that higher-twist corrections (HTs) are not needed in the analysis. As an aside, we should comment on the very large $x_{\mathbb{P}}$ domain. One cannot impose any cut at large $x_{\mathbb{P}}$, although, at present, there are essentially no diffractive DIS data available probing the $x_{\mathbb{P}}>10^{-1}$ domain. In addition to the cut on $Q^{2}$, we include the data with $M_{X}>2 \mathrm{GeV}$ in the fit.

Having discussed the details of kinematic cuts applied on the datasets in this analysis, we are now ready to discuss the corrections need to be taken into account for the $\mathrm{H} 1$ and ZEUS combined data. The first correction that one needs to apply on the datasets is due to the proton dissociation background. We should notice here that distinct methods have been used by the $\mathrm{H} 1$ and ZEUS experiments at HERA, and hence, the measured cross sections are not always presented with the corrections for proton dissociation backgrounds. In this analysis, the combined H1/ZEUS diffractive DIS cross sections are corrected by a global factor of 1.21 to account for the such contributions. Another correction one needs to consider comes from the fact that the all H1-LRG datasets used in our analysis are given for the range $|t|<$ $1 \mathrm{GeV}^{2}$ while the combined H1/ZEUS diffractive DIS cross sections, which is based upon proton-tagged samples, are restricted to the $t$ range of $0.09<|t|<0.55 \mathrm{GeV}^{2}$. Hence, one need to consider a global normalization factor to account this correction. The extrapolations for these two distinct range of $t$ have been performed by considering an exponential $t$ dependence of the inclusive diffractive DIS cross sections, using the $\mathrm{H} 1$ default value of exponential slope parameter $b=61 / \mathrm{GeV}^{2}$ [21,56]. Therefore, our analysis has been carried out by considering the above corrections as well as the preselection kinematical cuts applied on the analyzed datasets.

It is important to emphasize here that the list of diffractive DIS datasets considered in this work contains enough information to extract diffractive PDFs from a global QCD analysis. Clearly, there are other source of important processes that will provide additional information on the diffractive PDFs in the LHC era. Among these, one could consider diffractive dijet productions [57-63], providing useful information on the diffractive gluon density and, possibly, on the quark flavor separation respectively. As we discussed in the Introduction, the future high energy and high luminosity machines such as $\mathrm{LHeC}$ and FFC-eh will measure the diffractive DIS cross sections with the highest possible precision leading to the much better constraints for the diffractive PDFs [10]. Having discussed the diffractive DIS dataset as well as the kinematic cuts that we apply, we are now turn to discuss the method of $\chi^{2}$ minimizations and our approach to determine the diffractive PDFs uncertainties.

\section{THE METHOD OF $\chi^{2}$ MINIMIZATIONS AND DIFFRACTIVE PDFS UNCERTAINTIES}

In the present analysis, a $\chi^{2}(p)$ minimizations method to extract the fit parameters and an error calculation based on the "Hessian approach" to determine the uncertainties of diffractive PDFs are applied. As discussed in the literature review (e.g., see [64-66]), a precise understanding of uncertainties due to PDFs in a QCD analysis is crucial to precision studies of the standard model (SM) of particle physics [67], as well as to searches for new physics (NP) beyond the standard model (BSM) [68], especially for the future high-luminosity LHC and high-energy LHC. In turn, new measurements of SM processes from present or future high energy collider experiments such as HERA, LHC, Tevatron, RHIC, and $\mathrm{LHeC}$ can be used to constrain the uncertainties on the PDFs in a global QCD analysis. The most complete method for obtaining well constraints from the new datasets on the PDFs would be to add the up-to-date datasets into the global QCD analysis. Currently, the two most commonly used methods to estimate the PDFs uncertainties from a QCD analysis are the Monte Carlo approach $[69,70]$ and the standard Hessian method [39,71]. The details of Monte Carlo sampling techniques and use of neural networks (NN) as unbiased parametrization can be found in the analysis by the NNPDF Collaboration [4,52,72-74].

The details of the "Hessian approach" are fully addressed in Refs. [39,71,75-77]. In the standard "Hessian approach ," smaller number of " "error sets " are considered to obtain an estimate of the PDFs errors [71] in QCD fits. These error 
sets are correspond to the plus and minus eigenvectors directions in the space of PDFs fit parameters, which finally can be used to estimate the $\chi^{2}$ function near their global minimum. The Hessian approach relies on a quadratic approximation for the fit parameters dependence of the $\chi^{2}\left(\left\{\eta_{i}\right\}\right)$ minimization function and a linear approximation for the parameter dependence of the observables in question. In practice, the Hessian method works quite well for the most high energy observables in which used to determine the PDFs [39,71]. A version of this method, "Hessian profiling," has been included in the XFITTER package [78]. Our previous analysis on diffractive PDFs, GKG18-DPDF [17], was based on this package.

Let us discuss the $\chi^{2}\left(\left\{\eta_{i}\right\}\right)$ minimization procedure first. The goodness of fit is traditionally determined by the effective global $\chi_{\text {global }}^{2}\left(\left\{\eta_{i}\right\}\right)$ minimization algorithm that measures the quality of QCD fit between theory predictions and diffractive DIS experiments. This minimization strategy allow an extraction of independent parameters $\left\{\eta_{i}\right\}$ which specify the diffractive PDFs at the input scale $Q_{0}^{2}=2 \mathrm{GeV}^{2}$. This function is given by,

$$
\chi_{\text {global }}^{2}\left(\left\{\eta_{i}\right\}\right)=\sum_{n=1}^{N^{\exp }} w_{n} \chi_{n}^{2}\left(\left\{\eta_{i}\right\}\right),
$$

where $n$ labels a particular dataset and $w_{n}$ is a weight factor for the $n$th experiment with default value of $1[79,80]$. $\chi_{n}^{2}\left(\left\{\eta_{i}\right\}\right)$ can be written as

$$
\begin{aligned}
\chi_{n}^{2}\left(\left\{\eta_{i}\right\}\right)= & \left(\frac{1-\mathcal{N}_{n}}{\Delta \mathcal{N}_{n}}\right)^{2} \\
& +\sum_{j=1}^{N_{n}^{\text {data }}}\left(\frac{\mathcal{N}_{n} \mathcal{O}_{j}^{\exp }-\mathcal{T}_{j}^{\text {theory }}\left(\left\{\eta_{i}\right\}\right)}{\mathcal{N}_{n} \delta \mathcal{O}_{j}^{\exp }}\right)^{2} .
\end{aligned}
$$

The minimization of $\chi_{\text {global }}^{2}\left(\left\{\eta_{i}\right\}\right)$ function presented above is done applying the CERN program library MINUIT [81]. In Eq. (13), $\mathcal{O}$ represents the experimental measurement, and $\delta \mathcal{O}$ denotes the experimental uncertainty (statistical and systematic combined in quadrature), and $\mathcal{T}\left(\left\{\eta_{i}\right\}\right)$ is the theoretical value for the $i$ th experimental data point which depends on the input diffractive PDFs parameters $\left\{\eta_{i}\right\}$. In this equation, $\mathcal{N}_{n}$ is the overall normalization factors for a given data point of experiment $n$ and the $\Delta \mathcal{N}_{n}$ is the experimental normalization uncertainty. The (fitted) normalization factors $\mathcal{N}_{n}$ need to be extracted along with the diffractive PDFs parameters. We minimize the $\chi_{\text {global }}^{2}\left(\left\{\eta_{i}\right\}\right)$ value with the final 7 fit parameters. The obtained normalization factors $\mathcal{N}$ are presented in Table I for each experiment.

In order to show the effects arising from the use of the different diffractive DIS datasets, in Tables II-VI, we present the $\chi^{2}$ for each bin of $x_{\mathbb{P}}$ for our NLO QCD analysis. These
TABLE II. The values of $\chi^{2} / n^{\text {data }}$ for the H1/ZEUS Combined [21] data included in our QCD analysis. More detailed discussion of the description of the individual datasets, and the definitions of $\chi^{2}\left(\left\{\eta_{i}\right\}\right)$ are contained in the text.

H1/ZEUS Combined [21]

\begin{tabular}{lrrr}
$x_{\mathbb{P}}$ & $\chi^{2}(\mathrm{NLO})$ & $\chi^{2}(\mathrm{NNLO})$ & $n^{\text {data }}$ \\
\hline 0.0003 & & & 1 \\
0.0009 & 12.070 & 12.146 & 4 \\
0.0025 & 6.839 & 5.379 & 8 \\
0.0085 & 18.434 & 19.340 & 15 \\
0.016 & 19.611 & 20.401 & 17 \\
0.025 & 22.224 & 24.137 & 18 \\
0.035 & 9.985 & 10.019 & 19 \\
0.05 & 30.653 & 30.787 & 20 \\
0.075 & 13.359 & 12.787 & 19 \\
0.09 & 3.382 & 3.492 & 7 \\
& $\mathbf{1 3 3 . 4 0 1}$ & $\mathbf{1 3 5 . 1 7 9}$ & $\mathbf{1 2 8}$ \\
\hline \hline
\end{tabular}

TABLE III. The values of $\chi^{2} / n^{\text {data }}$ for the H1-LRG-12 [20] included in our QCD analysis. See the caption of Table II for further details.

\begin{tabular}{lccr}
\hline \hline & H1-LRG-12 [20] & & \\
$x_{\mathbb{P}}$ & $\chi^{2}(\mathrm{NLO})$ & $\chi^{2}(\mathrm{NNLO})$ & $n^{\text {data }}$ \\
\hline 0.0005 & 7.214 & 6.968 & 2 \\
0.001 & 14.145 & 15.073 & 19 \\
0.003 & 53.787 & 54.073 & 50 \\
0.01 & 26.936 & 26.128 & 62 \\
0.03 & 30.684 & 29.609 & 57 \\
& $\mathbf{1 3 2 . 7 6 8}$ & $\mathbf{1 3 2 . 4 9 9}$ & $\mathbf{1 8 8}$ \\
\hline \hline
\end{tabular}

TABLE IV. The values of $\chi^{2} / n^{\text {data }}$ for the H1-LRG-11 $\sqrt{s}=$ 225 [19] included in our QCD analysis. See the caption of Table II for further details.

\begin{tabular}{lccc}
\hline \hline & H1-LRG-11 $\sqrt{s}=225[19]$ & & \\
$x_{\mathbb{P}}$ & $\chi^{2}(\mathrm{NLO})$ & $\chi^{2}(\mathrm{NNLO})$ & $n^{\text {data }}$ \\
\hline 0.03 & 16.541 & 14.493 & 13 \\
& $\mathbf{1 6 . 5 4 1}$ & $\mathbf{1 4 . 4 9 3}$ & $\mathbf{1 3}$ \\
\hline \hline
\end{tabular}

TABLE V. The values of $\chi^{2} / n^{\text {data }}$ for the H1-LRG-11 $\sqrt{s}=$ 252 [19] included in our QCD analysis. See the caption of Table II for further details.

\begin{tabular}{lccr}
\hline \hline & H1-LRG-11 $\sqrt{s}=252[19]$ & & \\
$x_{\mathbb{P}}$ & $\chi^{2}(\mathrm{NLO})$ & $\chi^{2}(\mathrm{NNLO})$ & $n^{\text {data }}$ \\
\hline 0.0005 & 1.717 & 1.802 & 2 \\
0.003 & 15.951 & 15.446 & 10 \\
& $\mathbf{1 7 . 6 6 9}$ & $\mathbf{1 7 . 2 4 9}$ & $\mathbf{1 2}$ \\
\hline \hline
\end{tabular}


TABLE VI. The values of $\chi^{2} / n^{\text {data }}$ for the H1-LRG-11 $\sqrt{s}=$ 319 [19] included in our QCD analysis. See the caption of Table II for further details.

\begin{tabular}{lccc}
\hline \hline \multicolumn{4}{c}{ H1-LRG-11 $\sqrt{s}=319[19]$} \\
$x_{\mathbb{P}}$ & $\chi^{2}(\mathrm{NLO})$ & $\chi^{2}(\mathrm{NNLO})$ & $n^{\text {data }}$ \\
\hline 0.0005 & 0.073 & 0.229 & 2 \\
0.003 & 8.236 & 7.793 & 10 \\
& $\mathbf{8 . 3 0 9}$ & $\mathbf{8 . 0 2 2}$ & $\mathbf{1 2}$ \\
\hline \hline
\end{tabular}

tables illustrate the quality of our NLO and NNLO QCD fits to diffractive DIS cross sections datasets in terms of the individual $\chi^{2}$ values obtained for each experiment at a certain value of $x_{\mathbb{P}}$. As shown in these tables all the datasets are well fitted. We find $\chi^{2} /$ d.o.f $=312.07 / 346=0.91(\mathrm{NNLO})$ and $\chi^{2} /$ d.o.f $=310.83 / 346=0.89(\mathrm{NLO})$ which yield an acceptable fit to the experimental DIS data. The obtained $\chi^{2} /$ d.o.f shows a slightly improvement in the quality of the fit at NNLO accuracy.

In the following, we briefly discuss how to apply the "Hessian method" to determine the uncertainties of extracted diffractive PDFs. The basic procedure of this method is provided in Refs. [39,71,76,77]. The diffractive PDFs, $\beta \mathcal{F}\left(\beta, Q^{2} ; x_{\mathbb{P}}\right)$, defined at the initial scale $Q_{0}^{2}$, are parametrized by $\eta$ parameters. As we mentioned, the determination of the diffractive PDFs is obtained using a $\chi_{n}^{2}\left(\left\{\eta_{i}\right\}\right)$ function given in Eq. (13), which quantifies the discrepancy between the QCD theory predictions and the experimental observables of a global set of experiments, including the experimental errors.

In the well-known "Hessian" method one can diagonalize the covariance matrix and work in terms of the eigenvectors and eigenvalues. As we have mentioned earlier, the appropriate parameters set can be obtained by minimizing the $\chi_{n}^{2}\left(\left\{\eta_{i}\right\}\right)$ function. We entitled this as diffractive PDFs set $s_{0}$. The parameters values of $s_{0}$, i.e., $\left\{\eta_{1}^{0} \ldots \eta_{n}^{0}\right\}$, in which extracted from QCD fit to diffractive DIS data, will be presented and discussed in details in Sec. VI.

By moving away the parameters from their best fitted values, $\chi^{2}$ increases by the amount of $\Delta \chi^{2}$

$$
\begin{aligned}
\Delta \chi_{\text {global }}^{2} & =\chi_{\text {global }}^{2}(\{\eta\})-\chi_{0}^{2}\left(\left\{\eta^{0}\right\}\right) \\
& =\sum_{i, j=1}^{n}\left(\eta_{i}-\eta_{i}^{0}\right) H_{i j}\left(\eta_{j}-\eta_{j}^{0}\right),
\end{aligned}
$$

where $H_{i j}$ is the Hessian or error matrix which is given by

$$
H_{i j}=\left.\frac{1}{2} \frac{\partial^{2} \chi_{\text {global }}^{2}}{\partial \eta_{i} \partial \eta_{j}}\right|_{\text {min }} .
$$

These covariance or Hessian matrix can be obtained by running the CERN program library MINUIT [81]. Having ay hand the derivative of the observable $\mathcal{O}$ with respect to each parameter $\{\eta\}$, one can use the following equation

$$
\Delta \mathcal{O}^{2}=\Delta \chi_{\text {global }}^{2} \sum_{i, j=1}^{k} \frac{\partial \mathcal{O}}{\partial \eta_{i}} C_{i j} \frac{\partial \mathcal{O}}{\partial \eta_{j}}
$$

and can calculate the diffractive PDFs symmetric error bands as well as the corresponding observables such as the diffractive DIS cross sections for a desired values of confidence interval, $T=\Delta \chi_{\text {global }}^{2}$. We have to mentioned here that, in Eq. (16), $C_{i j} \equiv H_{i j}^{-1}$ is the elements of covariance or error matrix.

We should stressed that it is convenient to work in terms of the eigenvalues and orthogonal eigenvectors of covariance matrix which is given by

$$
\sum_{j=1}^{n} C_{i j} v_{j k}=\lambda_{k} v_{i k}
$$

The displacement of the parameter $\left\{\eta_{i}\right\}$ from its obtained minimum values $\eta_{i}^{0}$ can be expressed in terms of the rescaled eigenvectors $e_{i k}=\sqrt{\lambda_{k}} v_{i k}$. It reads

$$
\eta_{i}-\eta_{i}^{0}=\sum_{k=1}^{n} e_{i k} z_{k}
$$

Considering the orthogonality of eigenvectors $v_{i k}$ and putting Eq. (18) in (14), one can obtain

$$
\Delta \chi_{\text {global }}^{2}=\chi_{\text {global }}^{2}(\{\eta\})-\chi_{0}^{2}\left(\left\{\eta^{0}\right\}\right)=\sum_{k=1}^{n} z_{k}^{2} .
$$

The relevant neighborhood of $\chi^{2}$ is the interior of hypersphere with radius $T$. The uncertainty in the diffractive PDFs is then set by the requirement $\Delta \chi_{\text {global }}^{2}<T^{2}$ at some prescribed confidence level (CL). This means that

$$
\sum_{k=1}^{n} z_{k}^{2} \leq T^{2}
$$

Finally the neighborhood parameters can be written as

$$
\eta_{i}\left(s_{k}^{ \pm}\right)=\eta_{i}^{0} \pm t \sqrt{\lambda_{k}} v_{i k},
$$

with $s_{k}$ is the $k$ th set of diffractive PDFs, $t$ adapted to make the desired $T^{2}=\Delta \chi_{\text {global }}^{2}$ which is the tolerance for the required confidence interval (CL) and $t=T$ in the quadratic approximation.

Using the method discussed above, we accompany the construction of our QCD fit by reliable estimation of diffractive PDFs uncertainty. Finally uncertainty of a given observables $\mathcal{O}$ in the Hessian method, which can be the diffractive PDFs or related sort of diffractive observables, can calculate as $[39,71,77]$ 


$$
\Delta \mathcal{O}=\frac{1}{2}\left[\sum_{k=1}^{n}\left(\mathcal{O}\left(s_{k}^{+}\right)-\mathcal{O}\left(s_{k}^{-}\right)\right)^{2}\right]^{\frac{1}{2}} .
$$

In above equation, $\mathcal{O}\left(s_{k}^{+}\right)$and $\mathcal{O}\left(s_{k}^{-}\right)$are the value of observables $\mathcal{O}$ extracted from the input set of parameters $\eta_{i}\left(s_{k}^{ \pm}\right)$obtained from Eq. (21). The $\Delta \chi_{\text {global values }}^{2}$ determine the confidence region, and it is calculated so that the confidence level (CL) becomes the one- $\sigma$-error range for a given number of parameters. In this analysis, we calculate the diffractive PDFs uncertainty with $\Delta \chi_{\text {global }}^{2}=$ 1 and present their symmetric errors. We should mentioned here that a one-unit tolerance of $T=1$ would be the most appropriate choice if all the uncertainties in the data points as well as the theory would be Gaussian and perfectly accounted for. Hence, in order to obtain a more conservative error estimate one need to choose a reliable choice of tolerance.

For the case of $\eta$ degrees of freedom, the $\Delta \chi_{\text {global }}^{2}$ value needs to be calculated to determine the appropriate size of diffractive PDFs uncertainty. Assuming that the $\Delta \chi_{\text {global }}^{2}$ follows the $\chi^{2}$ distribution with $\eta$ degrees of freedom, we have the confidence level $\mathrm{P}$ as $[39,77]$

$$
\mathrm{P}=\int_{0}^{\Delta \chi_{\text {global }}^{2}} \frac{1}{2 \Gamma(\eta / 2)}\left(\frac{\xi}{2}\right)^{(\eta / 2)-1} e^{-\frac{\xi}{2}} d \xi
$$

where $\Gamma$ is the Gamma function. For the case of the one-freeparameter fit, we obviously have $\Delta \chi_{\text {global }}^{2}=1$. Since the diffractive PDFs in common QCD fits are considered with several free fit parameters, $\eta>1$, the value of $\Delta \chi_{\text {global }}^{2}$ should be calculated from Eq. (23). Considering the parameter number in our diffractive PDFs analysis, Eq. (23) leads to the tolerance criterion of $\Delta \chi_{\text {global }}^{2}=5.0$

In the next section, we will present full details of our fit quality and the extracted diffractive PDFs.

\section{RESULTS AND DISCUSSIONS}

In this section, we discuss the main results of this work, namely the NLO and NNLO diffractive PDFs sets extracted from QCD analyses of diffractive DIS datasets. We first discuss the numerical results of present work including the values of fitted parameters. Secondly, we discuss the quality of the QCD fits and compare our NLO and NNLO predictions to the fitted datasets. Then we show the resulting diffractive PDFs and their uncertainties. We mainly focus on the perturbative convergence upon applying the fracture function framework as well as inclusion of higher-order $\mathrm{QCD}$ corrections. Then we compare the extracted diffractive PDFs with the most recent results in literature, especially the results from GKG18-DPDF [17] analysis.

In Table VII, we present the fit parameters obtained in this work for our NLO and NNLO QCD analyses at the initial scale of $Q_{0}^{2}=2 \mathrm{GeV}^{2}$. Values marked with (*) are fixed in
TABLE VII. Best fit parameters obtained in HK19-DPDF NLO and NNLO QCD fits at the initial scale of $Q_{0}^{2}=2 \mathrm{GeV}^{2}$ and their experimental uncertainties. Values marked with $\left(^{*}\right)$ are fixed in the fit since the analyzed diffractive DIS datasets do not constrain these parameters well enough.

\begin{tabular}{lcc}
\hline \hline Parameters & NLO & NNLO \\
\hline $\mathcal{N}_{q}$ & $0.0005 \pm 0.0002$ & $0.0023 \pm 0.0008$ \\
$\alpha_{q}$ & $-0.3334 \pm 0.0388$ & $-0.1298 \pm 0.030$ \\
$\beta_{q}$ & $0.6157 \pm 0.03264$ & $0.7308 \pm 0.032$ \\
$\gamma_{q}$ & $-0.663^{*}$ & $-1.647^{*}$ \\
$\eta_{q}$ & $89.327^{*}$ & $28.959^{*}$ \\
$\mathcal{N}_{g}$ & $0.2075 \pm 0.01027$ & $0.3265 \pm 0.018$ \\
$\alpha_{g}$ & $0.4144 \pm 0.03791$ & $0.662 \pm 0.0523$ \\
$\beta_{g}$ & $0.50^{*}$ & $0.50^{*}$ \\
$\gamma_{g}$ & $-0.0366^{*}$ & $-0.4200^{*}$ \\
$\eta_{g}$ & $0.0^{*}$ & $0.0^{*}$ \\
$w_{1}$ & $-1.1912 \pm 0.00611$ & $-1.1969 \pm 0.0063$ \\
$w_{2}$ & $0.0^{*}$ & $0.0^{*}$ \\
$w_{3}$ & $86.156^{*}$ & $82.532^{*}$ \\
$w_{4}$ & $1.7735 \pm 0.02647$ & $1.7441 \pm 0.02595$ \\
$\alpha_{s}\left(M_{Z}^{2}\right)$ & $0.1185^{*}[49,50,72]$ & $0.1185^{*}[49,50,72]$ \\
$m_{c}$ & $1.51^{*}[49,50,72]$ & $1.51^{*}[49,50,72]$ \\
$m_{b}$ & $4.92^{*}[49,50,72]$ & $4.92^{*}[49,50,72]$ \\
$\chi^{2} /$ d.o.f & $312.07 / 346=0.91$ & $310.83 / 346=0.89$ \\
\hline \hline
\end{tabular}

the fit since the analyzed datasets do not constrain these parameters well enough. These fitted parameters include $\{\mathcal{N}, \alpha, \beta, \gamma, \eta\}$ for the diffractive quark and gluon densities. The parameters for the weight factor $\mathcal{W}\left(x_{\mathbb{P}}\right)$ also presented as well. As one can see from this table, the values of the physical parameters used in the computation of diffractive DIS cross section and in the evolution of diffractive PDFs are the same as those used in the NNPDF global analysis of unpolarized PDFs and FFs [49,50,72]. Specifically, we use $\alpha_{s}\left(M_{Z}^{2}\right)=$ 0.1185 as reference value for the QCD couplings, and $m_{c}=$ 1.51 and $m_{b}=4.92 \mathrm{GeV}$ for the charm- and bottom-quark masses.

In the present fit of diffractive PDFs, the analyzed datasets is much more limited than in a typical global proton PDFs fit. As we discussed earlier, diffractive DIS data allow for the determination of only two independent combinations of densities, namely the singlet and the gluon. In addition, these distributions at some region of $\beta$ sill remain unconstrained. This effect is more enhanced for the case of diffractive gluon density, due to the reduced sensitivity of the diffractive DIS data included in our QCD fit to the gluon distribution. Therefore one would expect that the diffractive gluon PDFs is determined with larger uncertainties than the diffractive quark PDFs. This issue deserves a separate comment, which we will discuss later. In addition, diffractive DIS is blind to the separation between quark and antiquark distributions. These points confirm that the input functional form and its sensitivity to the parameter space of $\left\{\eta_{i}\right\}$ and $\left\{w_{i}\right\}$ need to be clearly 


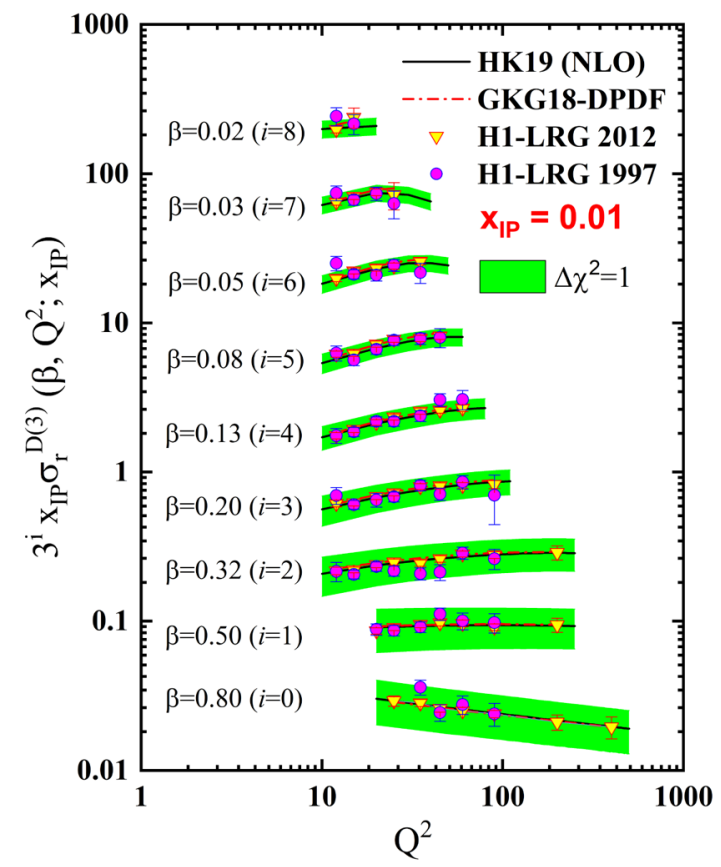

FIG. 3. The NLO theory predictions for the diffractive reduced cross sections $x_{\mathbb{P}} \sigma_{r}^{D(3)}\left(\beta, Q^{2} ; x_{\mathbb{P}}\right)$ as a function of $\mathrm{Q}^{2}$ for some selected values of $\beta$ and for $x_{\mathbb{P}}=0.01$. To facilitate the graphical presentation, we have plotted $3^{i} \times x_{\mathbb{P}} \sigma_{r}^{D(3)}$ with $i$ indicated in parentheses in the figure. The NLO theory predictions are compared with the H1-LRG-2012 [20] and H1-LRG-1997 [15] measurements. The NLO theory prediction based on the GKG18-DPDF [17] also have been shown for comparison.

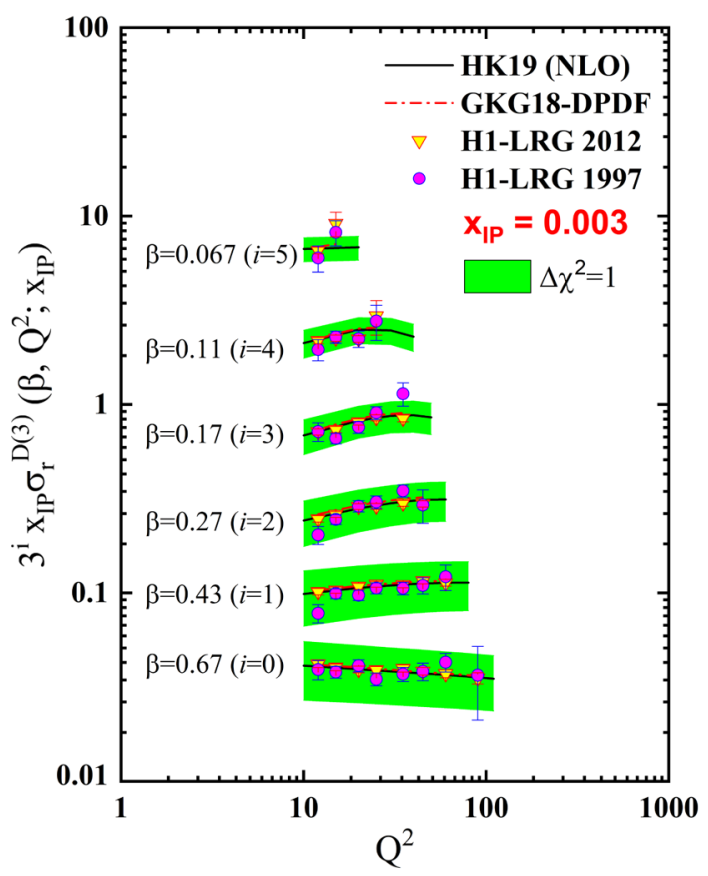

FIG. 4. The NLO theory predictions for the diffractive reduced cross sections $x_{\mathbb{P}} \sigma_{r}^{D(3)}\left(\beta, Q^{2} ; x_{\mathbb{P}}\right)$ for $x_{\mathbb{P}}=0.003$. See the caption of Fig. 3 for further details. investigated. As we mentioned, the currently available diffractive DIS datasets do not fully constrain the entire $\beta$ and $x_{\mathbb{P}}$ dependence of quark and gluon diffractive PDFs presented in Eq. (11). Consequently, we are forced to make some restrictions on the parameter space of $\left\{\eta_{i}\right\}$ and $\left\{w_{i}\right\}$. For the diffractive gluon density, we set $\eta_{g}$ to 0 and $\beta_{g}$ to 0.5 . These only marginally limit the freedom in the input functional form for the gluon density. We fixed the $\gamma_{q}, \eta_{q}$, and $\gamma_{g}$ to their best fit values. The lack of diffractive DIS data at high- $x_{\mathbb{P}}$, mean that the flux factor is not really well determined in this region. Hence, for the flux factor, we set the $w_{2}$ to 0 and fixed other variable $\left\{w_{3}\right\}$ to their best fit values. In total, these leave us with 7 free parameters in our QCD fit (three for quarks, two for the gluon density and two for the flux factor), which we include later on our diffractive PDFs uncertainty estimations.

In the following, we discuss the overall fit quality. Figures 3 and 4 show the NLO theory predictions based on the extracted diffractive PDFs for the diffractive reduced

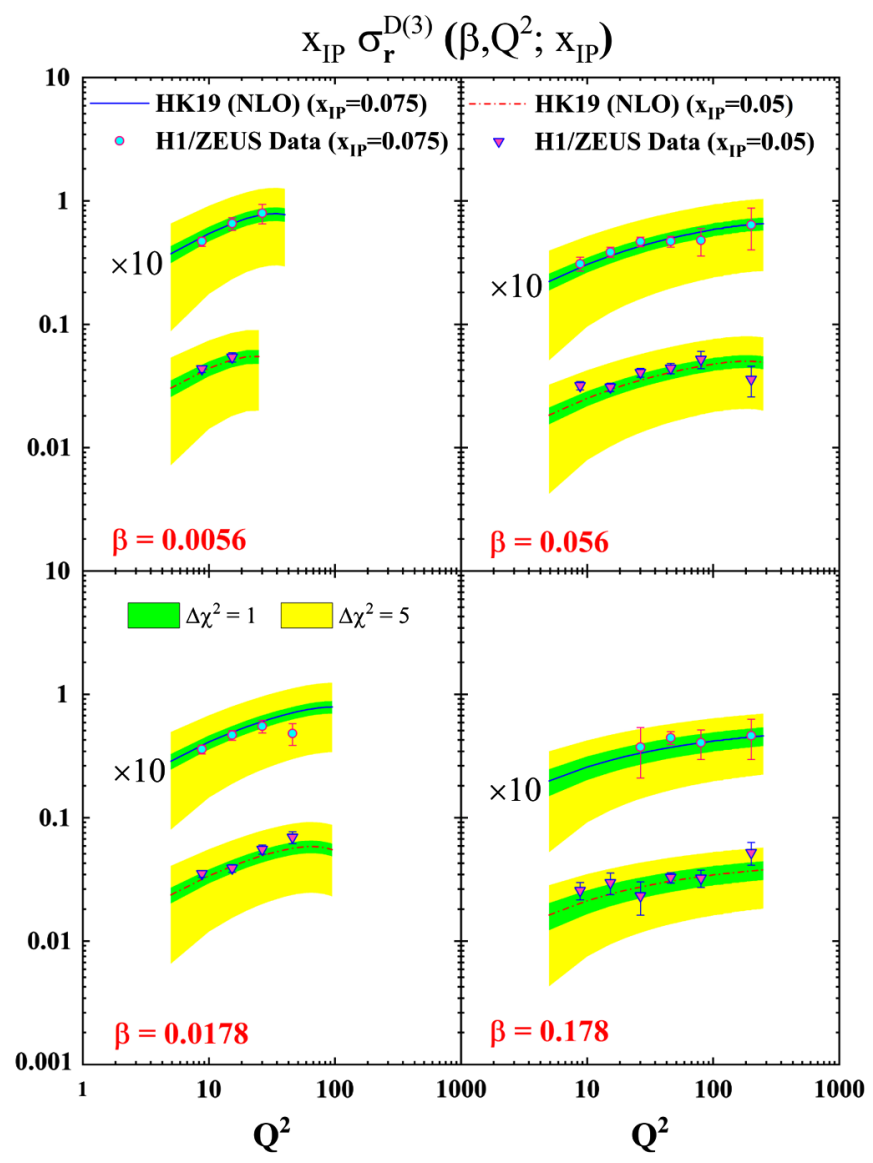

FIG. 5. The NLO theory predictions for the diffractive reduced cross sections $x_{\mathbb{P}} \sigma_{r}^{D(3)}\left(\beta, Q^{2} ; x_{\mathbb{P}}\right)$ as a function of $\mathrm{Q}^{2}$ for some selected values of $\beta$ and for two representative bins of $x_{\mathbb{P}}=0.05$ and 0.075. The H1/ZEUS combined [21] diffractive DIS measurements also has been shown for comparison. The error bands correspond to the fit uncertainties for the choice of tolerance $\Delta \chi^{2}=1$ and $\Delta \chi^{2}=5$. 
cross sections $x_{\mathbb{P}} \sigma_{r}^{D(3)}\left(\beta, Q^{2} ; x_{\mathbb{P}}\right)$ for two representative bins of $x_{\mathbb{P}}=0.01$ and 0.003 . The uncertainty bands correspond to the choice of tolerance $T=\Delta \chi_{\text {global }}^{2}=1$ also have been shown as well. These error bands represent the fit uncertainties derived only from the experimental input. In order to judge the fit quality, our NLO theory predictions are compared with the H1-LRG-2012 [20] measurements. It would be also interesting to examine the fit quality in comparison to the datasets that we did not include in our fit such as old diffractive DIS data measurements at HERA. Hence, in Figs. 3 and 4, we also compare our NLO theory predictions with the H1-LRG1997 [15] measurements. As one can see, prediction based on our diffractive PDFs sets are in reasonably good agreements with the HERA data. The NLO theory prediction based on the GKG18-DPDF [17] also have been shown for comparison. From the theory predictions presented in these figures one can conclude that the scale dependence induced by the evolution equations of Eq. (7) is perfectly consistent with the diffractive DIS data. The results clearly indicate that one can use the fracture functions approach to describe diffractive DIS in perturbative QCD at the kinematic region covered by the $e p$ collider HERA as well as other hadron colliders.

The previous figures show the quality of the description of the H1-LRG-2012 data with our NLO theory predictions. It would be interesting to observe that the fit quality to the inclusive $\mathrm{H} 1$ and ZEUS combined measurement for the inclusive diffractive DIS cross sections. In the following we discuss the overall fit quality of this dataset. For completeness, in Fig. 5, the NLO theory predictions for the diffractive reduced cross sections $x_{\mathbb{P}} \sigma_{r}^{D(3)}\left(\beta, Q^{2} ; x_{\mathbb{P}}\right)$ have been presented as a function of $\mathrm{Q}^{2}$ for some selected values of $\beta$ and for two representative bins of $x_{\mathbb{P}}=0.05$ and 0.075. The H1/ZEUS combined [21] diffractive DIS measurements also has been shown for comparison. The uncertainty bands for HK19-DPDF analysis as well as for the GKG18-DPDF are correspond to the choice of tolerance $T=\Delta \chi_{\text {global }}^{2}=1$ and $T=\Delta \chi_{\text {global }}^{2}=5$. These results show that the quality of the description between our NLO theory predictions and all the H1/ZEUS combined data points analyzed in this study is quite acceptable.
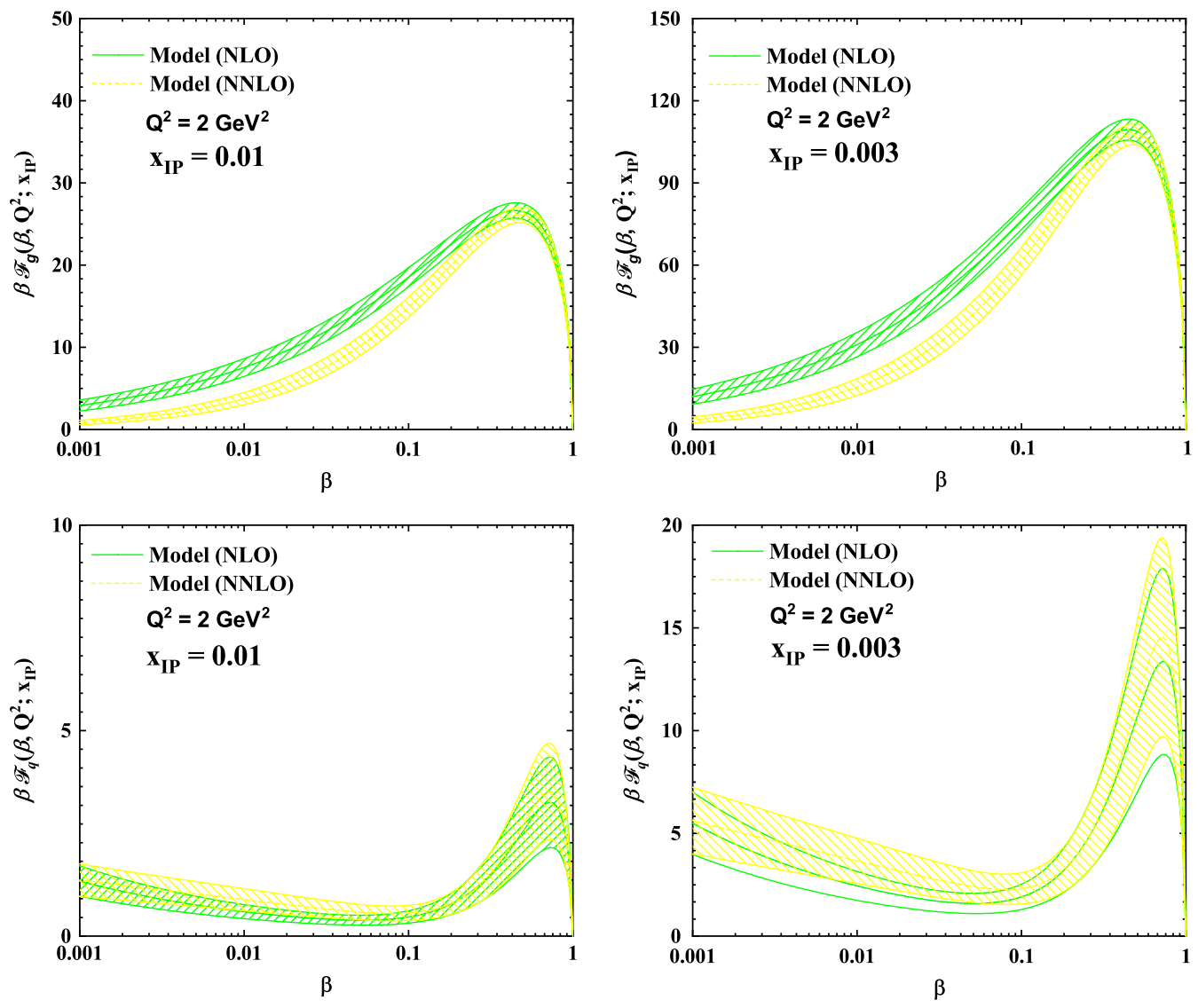

FIG. 6. Comparison the NLO and NNLO quark $\beta \mathcal{F}_{q}\left(\beta, Q^{2} ; x_{\mathbb{P}}\right)$ and gluon $\beta \mathcal{F}_{g}\left(\beta, Q^{2} ; x_{\mathbb{P}}\right)$ diffractive PDFs at the input scale $\mathrm{Q}_{0}^{2}=2 \mathrm{GeV}^{2}$ for two different $x_{\mathbb{P}}$ bin of 0.01 and 0.003 . The uncertainty bands of diffractive PDFs presented for the choice of tolerance $T=\Delta \chi_{\text {global }}^{2}=1$ for the $68 \%$ (one-sigma) confidence level (CL). 
In the rest of this section, we present the resulting diffractive PDFs at NLO and NNLO accuracy. We also compare the HK19-DPDF results with the most recent analysis of GKG18-DPDF [17]. We mainly discuss the main difference of the results as well as the effect arising from including the higher order $\mathrm{QCD}$ corrections. We now compare the diffractive $\mathrm{PDFs} \beta \mathcal{F}\left(\beta, Q^{2} ; x_{\mathbb{P}}\right)$ obtained from this analysis with other results in literature, and discuss how their central values and uncertainties vary. As we presented in Sec. V, this analysis is based on a standard "parameterfitting" criterion, and we plan to present the uncertainty bands of diffractive PDFs considering the choice of tolerance $T=\Delta \chi_{\text {global }}^{2}=1$ for the $68 \%$ (one-sigma) confidence level (CL) uncertainty.

In the following, we now turn to discuss the obtained diffractive PDFs and their uncertainties. In Fig. 6 detailed comparisons of the NLO and NNLO diffractive quark $\beta \mathcal{F}_{q}\left(\beta, Q^{2} ; x_{\mathbb{P}}\right)$ and gluon $\beta \mathcal{F}_{g}\left(\beta, Q^{2} ; x_{\mathbb{P}}\right)$ PDFs have been presented at the input scale $\mathrm{Q}_{0}^{2}=2 \mathrm{GeV}^{2}$ for two different $x_{\mathbb{P}}$ bin of 0.01 and 0.003 . The uncertainty bands of diffractive PDFs presented for the choice of tolerance $T=$ $\Delta \chi_{\text {global }}^{2}=1$ for the $68 \%$ (one-sigma) confidence level (CL). In order to study the perturbative convergence of the diffractive PDFs upon inclusion of higher order QCD corrections, in Fig. 6 we also compare our NLO and NNLO determinations among each other. Comparing our results, it can be seen that the NLO and NNLO quark diffractive PDFs $\beta \mathcal{F}_{q}$ are similar in size for all range of $\beta$. However a small difference can be seen in range of $\beta<0.1$ for the $x_{\mathbb{P}}=0.003$. The single most striking observation to emerge from the NLO and NNLO comparisons is for the case of the diffractive gluon PDFs $\beta \mathcal{F}_{g}$. One can see that the diffractive gluon PDFs is affected by including the higher-order QCD corrections. Concerning the shapes of the diffractive gluon PDFs, a number of interesting differences between the NLO and NNLO results can be seen from the comparisons in Fig. 6. Significant differences in shape are observed for the small values of $\beta$, especially for the range of $\beta<0.2$. In this range, the diffractive gluon PDFs at NLO accuracy have a larger magnitude than the NNLO results.

As one can see the NLO and NNLO uncertainties are similar in size showing that the inclusion of higher-order QCD corrections do not improve the uncertainty. As we presented in Tables II-VI, concerning the fit quality of the total diffractive DIS dataset, the most noticeable feature is the improvement upon inclusion of higher-order corrections. However, the improvement of the total $\chi^{2} /$ d.o.f is not significant when going from NLO to NNLO. This demonstrates that the inclusion of the NNLO QCD corrections could slightly improves the fit quality as well as the description of the data.

In Fig. 7, we present the quark $\beta \mathcal{F}_{q}\left(\beta, Q^{2} ; x_{\mathbb{P}}\right)$ and gluon $\beta \mathcal{F}_{g}\left(\beta, Q^{2} ; x_{\mathbb{P}}\right)$ diffractive PDFs at the scale of
$\mathrm{Q}^{2}=100 \mathrm{GeV}^{2}$ and for the $x_{\mathbb{P}}=0.01$. Comparing these two results, it can be seen that the differences between the NLO and NNLO sets are very small, both for central values and uncertainties.

We are in a position to compare our best-fit NLO diffractive PDFs to their counterparts in the GKG18DPDF analysis [17] which applied the general method used to extract diffractive PDFs from available data by considering a number of assumptions motivated by the Regge phenomenology. In Fig. 8, we present the results for the diffractive gluon and quark PDFs at $\mathrm{Q}^{2}=10 \mathrm{GeV}^{2}$ for two selected bin of $x_{\mathbb{P}}=0.01$ and 0.003 . We see that the overall effects of the new methodology are comparable to those reported by [17], but that they act in different kinematical regions of $\beta$ and $x_{\mathbb{P}}$ and for different diffractive PDFs. From Fig. 8, it is clear that central values move very little while diffractive PDFs uncertainties are slightly increased. For instance, for the light quarks and the gluon diffractive PDFs the impact of the new methodology mainly effect the small regions of $\beta ; \beta<0.1$, where it produces an enhancement for the gluon density and a reduction for the quark density. We should emphasize here that the QCD analysis of diffractive PDFs motivated by the Regge phenomenology uses the GRV parametrization

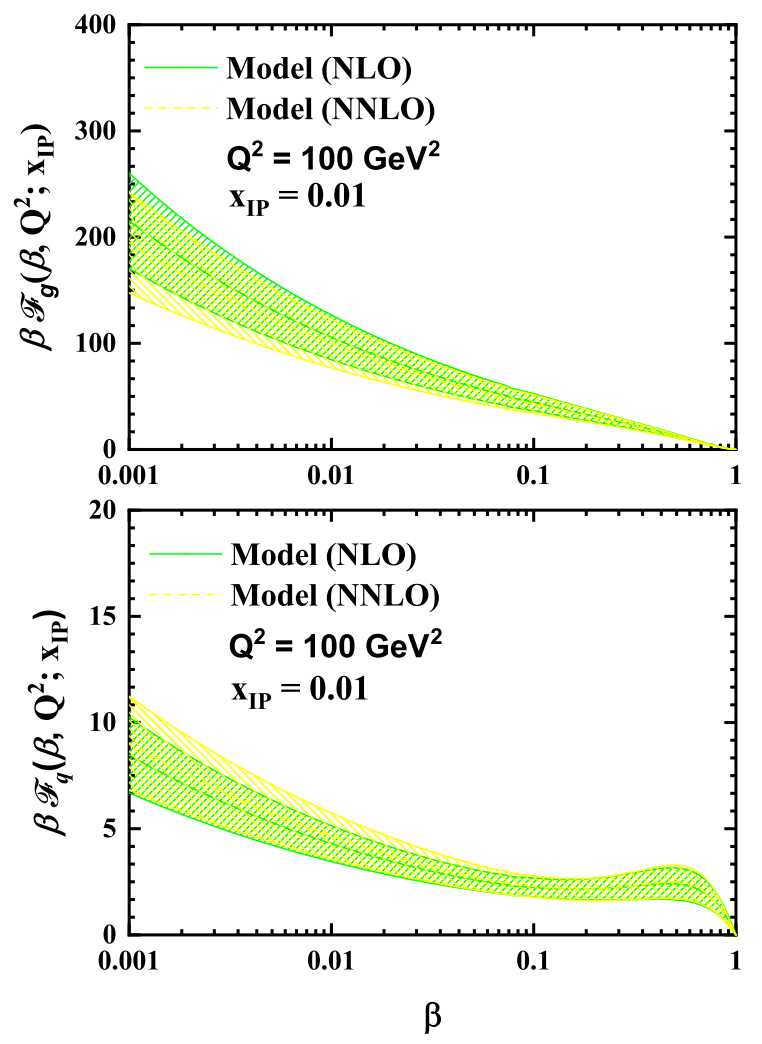

FIG. 7. Comparison the NLO and NNLO quark $\beta \mathcal{F}_{q}\left(\beta, Q^{2} ; x_{\mathbb{P}}\right)$ and gluon $\beta \mathcal{F}_{g}\left(\beta, Q^{2} ; x_{\mathbb{P}}\right)$ diffractive PDFs at $\mathrm{Q}^{2}=100 \mathrm{GeV}^{2}$ for $x_{\mathbb{P}}=0.01$. The uncertainty bands of diffractive PDFs presented for the choice of tolerance $T=\Delta \chi_{\text {global }}^{2}=1$. 

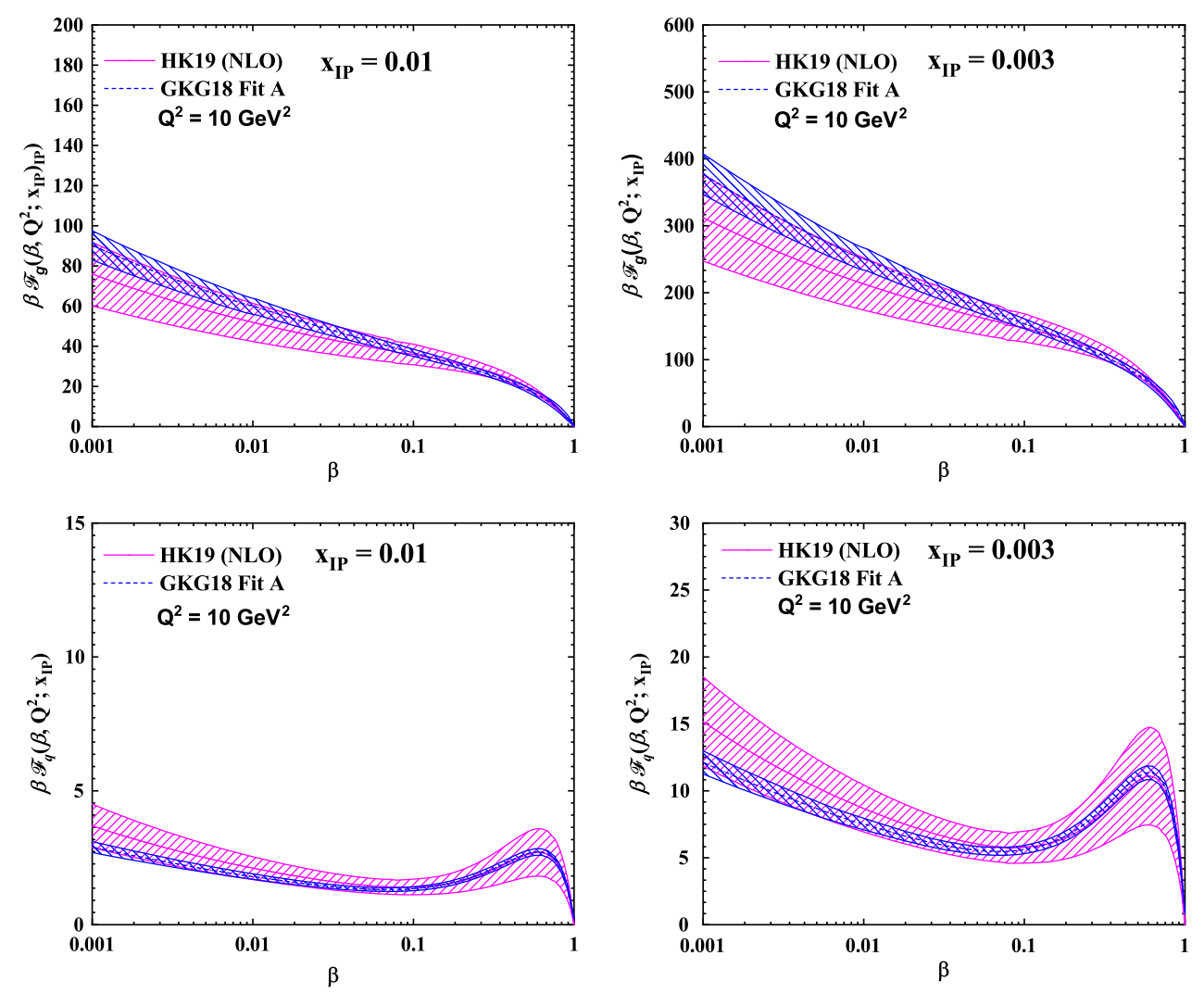

FIG. 8. Comparison between our NLO quark and gluon diffractive PDFs and the results by GKG18-DPDF [17]. We show results for the diffractive gluon and quark PDFs at $\mathrm{Q}^{2}=10 \mathrm{GeV}^{2}$ for two selected bin of $x_{\mathbb{P}}=0.01$ and 0.003 . The uncertainty bands for HK19-DPDF results as well as for the GKG18-DPDF analysis are correspond to the choice of tolerance $T=\Delta \chi_{\text {global }}^{2}=1$.

derived from a fit to pion structure function data [82] for the Reggeon parton density, and hence, an additional source of uncertainty needs to be taken into account in presenting the diffractive PDFs in the kinematic space of $z, \mathrm{Q}^{2}$ and $x_{\mathbb{P}}$. Overall, there is satisfactory agreement between the two methodology of diffractive PDFs determinations. Hence, this study strengthens the idea of using the fracture function approach to determine diffractive PDFs from a QCD analysis of diffractive DIS datasets.

Let us conclude this section with a presentation of heavy quark diffractive PDFs obtained in this study. As we discussed in Sec. II C, for the calculation of heavy-quark structure functions is performed in the FONLL GM-VFNS [46]. In Fig. 9, the charm $\beta c\left(\beta, Q^{2} ; x_{\mathbb{P}}\right)$ and bottom $\beta b\left(\beta, Q^{2} ; x_{\mathbb{P}}\right)$ quark diffractive PDFs obtained from our NLO QCD fits have been shown at $\mathrm{Q}^{2}=100 \mathrm{GeV}^{2}$ and for $x_{\mathbb{P}}=0.01$. The error bands shown in these figures correspond to the fit uncertainties derived only from the experimental input. The results from GKG18-DPDF analysis [17] are also presented for comparison. As one can see from these results, the overall agreements are well and only small differences between our NLO results and
GKG18-DPDF can be found for all heavy quark diffractive PDFs at lower values of $\beta ; \beta<0.02$.

Through our results presented in section, we have shown that our QCD analyses show good agreements with the results obtained by GKG18-DPDF parametrization. In addition, we found that our theory predictions based on the extracted diffractive PDFs are in satisfactory agreements with the $\mathrm{H} 1$ and ZEUS diffractive DIS datasets over a wide range of DIS kinematics. The scale dependence of these datasets is found to be in satisfactory agreement with the one predicted for the fracture function, driven by the DGLAP evolution equations. As a short summary, the results of this research support the idea of extracting the diffractive PDFs using the fracture functions approach. The presented QCDbased predictions of diffractive DIS processes also provide an important step towards an improved understanding of such processes and also represent a precise test of the employed theoretical concepts to extract PDFs from a QCD analysis of diffractive DIS observables. In particular, it is observed in this analysis, that for the given kinematical range of the HERA hard diffraction events, higher-order QCD corrections are of crucial importance. 

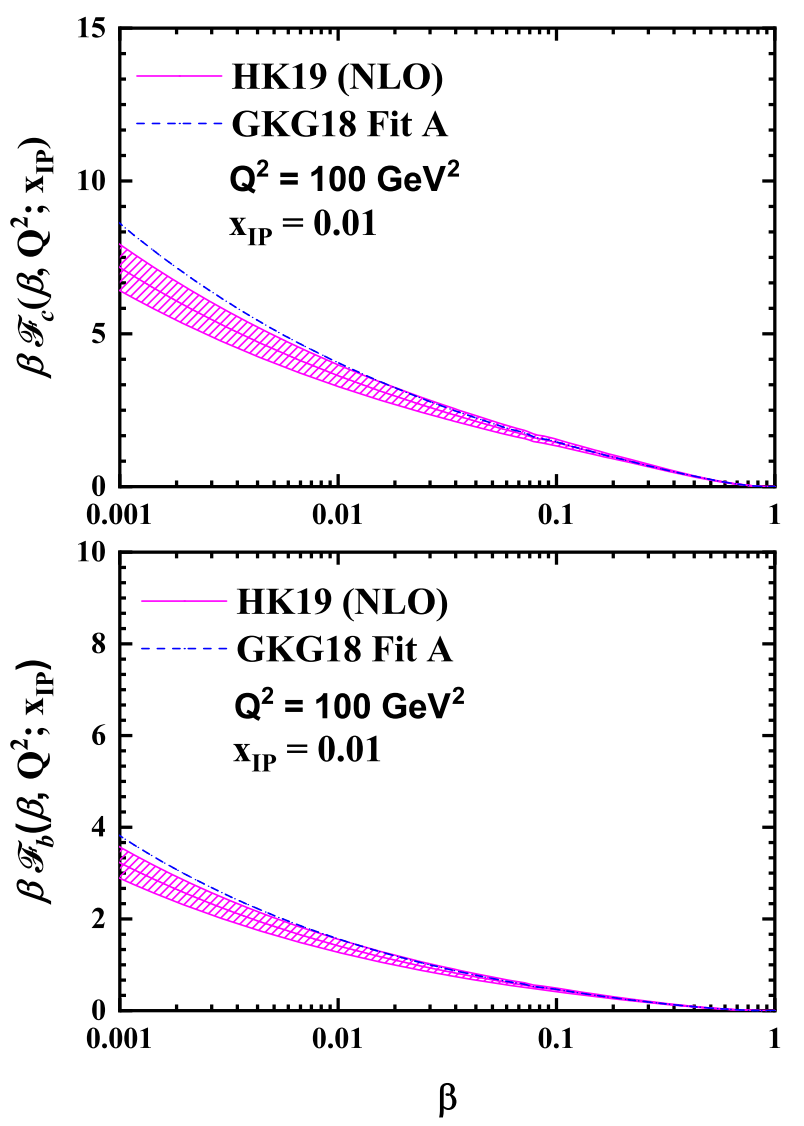

FIG. 9. $\quad$ Charm $\beta \mathcal{F}_{c}\left(\beta, Q^{2} ; x_{\mathbb{P}}\right)$ and bottom $\beta \mathcal{F}_{b}\left(\beta, Q^{2} ; x_{\mathbb{P}}\right)$ quark diffractive PDFs obtained from our NLO QCD fits at $\mathrm{Q}^{2}=$ $100 \mathrm{GeV}^{2}$ and for $x_{\mathbb{P}}=0.01$. The results from GKG18-DPDF [17] analysis also presented for comparison. The uncertainty bands are correspond to the choice of tolerance $T=\Delta \chi_{\text {global }}^{2}=1$.

\section{SUMMARY AND CONCLUSIONS}

In summary, in this work, we have presented a set of diffractive PDFs at next-to-leading order (NLO) and next-to-next-leading order (NNLO) accuracy obtained from the most up-to-date diffractive DIS data including the most recent combined datasets from $\mathrm{H} 1$ and ZEUS Collaborations. The new combined diffractive DIS data from run II at HERA allow for a very accurate determination of the quark and gluon distributions in a wide range of scaled fractional momentum $\beta$ and longitudinal momentum fractions $x_{\mathbb{P}}$. We supplement our best-fit diffractive PDFs parameterizations with the reliable uncertainties obtained according to the "Hessian approach" which allows the experimental uncertainties to propagate to an arbitrary observable such as diffractive DIS cross sections. The theory predictions for the hard-scattering diffractive DIS processes in this analysis maintain the NLO accuracy, and for the first time the NNLO accuracy in QCD and employ the FONLL GM-VNFS, which has been shown to provide an excellent description of the existing diffractive DIS data.

In addition to the points mentioned, the theory framework applied in this analysis features a number of new improvements. We work in the framework of fracture functions, as a new method to extract diffractive PDFs inspired by the fully factorization theorem for diffractive DIS processes, which allows a good description of diffractive DIS cross sections. We have demonstrated that diffractive DIS is consistent within this picture and one can introduced diffractive PDFs accordingly. We have shown that a simple parametrization form for the diffractive PDFs along with the fully factorized approach for the cross section provide very accurate descriptions of the diffractive DIS datasets measured by $\mathrm{H} 1$ and ZEUS collaborations at HERA. The diffractive PDFs extracted for a QCD analysis in the fracture functions are also in satisfactory agreements by other analysis in literature. Finally, our results verify that the scale dependence of the data agrees well with the one predicted by the fracture function formalism. Hence, we can conclude that in the diffractive DIS kinematics analyzed in this study, the fracture functions framework can provide a good understanding of the physical picture of this sort of high energy processes.

Our analysis can be extended in various different directions. First, our analysis can be extended to the diffractive dijet events at HERA [62,63]. For the future, our main goal is to assess the impact of diffractive dijet productions data on the diffractive PDFs and their uncertainties. More detailed discussions on this new extraction of diffractive PDFs at NLO accuracy will be presented in our next study. Second, in terms of future work, it would be interesting to repeat the analysis described here and present a combined QCD analysis of recent datasets measured by the $\mathrm{H} 1$ and ZEUS collaborations at HERA on the diffractive DIS and leading-nucleon productions [83-85]. The LO, NLO and NNLO diffractive PDFs sets $\beta \mathcal{F}\left(\beta, Q^{2} ; x_{\mathbb{P}}\right)$ presented in this work are available in the LHAPDF format [86] from the author upon request.

\section{ACKNOWLEDGMENTS}

The author is especially grateful to Muhammad Goharipour, Maryam Soleymaninia and S. Atashbar Tehrani for carefully reading the manuscript and fruitful discussions. Author thanks University of Science and Technology of Mazandaran, and School of Particles and Accelerators, Institute for Research in Fundamental Sciences (IPM) for financial support of this project. 
[1] J. Gao, L. Harland-Lang, and J. Rojo, The structure of the proton in the LHC precision era, Phys. Rep. 742, 1 (2018).

[2] P. Newman and M. Wing, The hadronic final state at HERA, Rev. Mod. Phys. 86, 1037 (2014).

[3] H. Abramowicz et al. (H1 and ZEUS Collaborations), Combination and QCD analysis of charm and beauty production cross-section measurements in deep inelastic ep scattering at HERA, Eur. Phys. J. C 78, 473 (2018).

[4] R. D. Ball et al. (NNPDF Collaboration), Parton distributions for the LHC Run II, J. High Energy Phys. 04 (2015) 040.

[5] L. A. Harland-Lang, A. D. Martin, P. Motylinski, and R. S. Thorne, Parton distributions in the LHC era: MMHT 2014 PDFs, Eur. Phys. J. C 75, 204 (2015).

[6] A. Accardi, L. T. Brady, W. Melnitchouk, J. F. Owens, and N. Sato, Constraints on large- $x$ parton distributions from new weak boson production and deep-inelastic scattering data, Phys. Rev. D 93, 114017 (2016).

[7] M. Klein, Future deep inelastic scattering with the LHeC, arXiv:1802.04317.

[8] M. Mangano et al., Future circular collider: Vol. 1 Physics opportunities, Report No. CERN-ACC-2018-0056.

[9] M. Benedikt et al., Future circular collider: Vol. 3 The Hadron Collider (FCC-hh), Report No. CERN-ACC-20180058.

[10] N. Armesto, P. R. Newman, W. Slominski, and A. M. Stasto, Inclusive diffraction at the $\mathrm{LHeC}$ and FCC-eh, arXiv: 1901.09076 .

[11] J. C. Collins, Proof of factorization for diffractive hard scattering, Phys. Rev. D 57, 3051 (1998); Erratum, Phys. Rev. D61, 019902(E) (1999).

[12] G. Ingelman and P. E. Schlein, Jet structure in high mass diffractive scattering, Phys. Lett. 152B, 256 (1985).

[13] I. Helenius and C. O. Rasmussen, Hard diffraction in photoproduction, arXiv:1901.05261.

[14] C. O. Rasmussen and T. Sjöstrand, Models for total, elastic and diffractive cross sections, Eur. Phys. J. C 78, 461 (2018).

[15] A. Aktas et al. (H1 Collaboration), Measurement and QCD analysis of the diffractive deep-inelastic scattering cross-section at HERA, Eur. Phys. J. C 48, 715 (2006).

[16] S. Chekanov et al. (ZEUS Collaboration), A QCD analysis of ZEUS diffractive data, Nucl. Phys. B831, 1 (2010).

[17] M. Goharipour, H. Khanpour, and V. Guzey, First global next-to-leading order determination of diffractive parton distribution functions and their uncertainties within the xFitter framework, Eur. Phys. J. C 78, 309 (2018).

[18] D. Britzger, J. Currie, T. Gehrmann, A. Huss, J. Niehues, and R. Žleb'ik, Dijet production in diffractive deep-inelastic scattering in next-to-next-to-leading order QCD, Eur. Phys. J. C 78, 538 (2018).

[19] F. D. Aaron et al. (H1 Collaboration), Measurement of the diffractive longitudinal structure function $F_{L}^{D}$ at HERA, Eur. Phys. J. C 72, 1836 (2012); 71, 1836 (2011).

[20] F. D. Aaron et al. (H1 Collaboration), Inclusive measurement of diffractive deep-inelastic scattering at HERA, Eur. Phys. J. C 72, 2074 (2012).
[21] F. D. Aaron et al. (H1 and ZEUS Collaborations), Combined inclusive diffractive cross sections measured with forward proton spectrometers in deep inelastic ep scattering at HERA, Eur. Phys. J. C 72, 2175 (2012).

[22] L. Trentadue and G. Veneziano, Fracture functions: An improved description of inclusive hard processes in QCD, Phys. Lett. B 323, 201 (1994).

[23] D. de Florian and R. Sassot, QCD analysis of diffractive and leading proton DIS structure functions in the framework of fracture functions, Phys. Rev. D 58, 054003 (1998).

[24] F. A. Ceccopieri, QCD analysis of forward neutron production in DIS, Eur. Phys. J. C 74, 3029 (2014).

[25] S. Shoeibi, H. Khanpour, F. Taghavi-Shahri, and K. Javidan, Determination of neutron fracture functions from a global QCD analysis of the leading neutron production at HERA, Phys. Rev. D 95, 074011 (2017).

[26] S. Shoeibi, F. Taghavi-Shahri, H. Khanpour, and K. Javidan, Phenomenology of leading nucleon production in ep collisions at HERA in the framework of fracture functions, Phys. Rev. D 97, 074013 (2018).

[27] F. A. Ceccopieri, Single-diffractive Drell-Yan pair production at the LHC, Eur. Phys. J. C 77, 56 (2017).

[28] J. C. Collins, Factorization in hard diffraction, J. Phys. G 28, 1069 (2002).

[29] M. Grazzini, L. Trentadue, and G. Veneziano, Fracture functions from cut vertices, Nucl. Phys. B519, 394 (1998).

[30] J. A. M. Vermaseren, A. Vogt, and S. Moch, The third-order QCD corrections to deep-inelastic scattering by photon exchange, Nucl. Phys. B724, 3 (2005).

[31] A. D. Martin, M. G. Ryskin, and G. Watt, A QCD analysis of diffractive deep-inelastic scattering data, Eur. Phys. J. C 37, 285 (2004).

[32] A. D. Martin, M. G. Ryskin, and G. Watt, Diffractive parton distributions from perturbative QCD, Eur. Phys. J. C 44, 69 (2005).

[33] S. T. Monfared, A. N. Khorramian, and S. A. Tehrani, A global analysis of diffractive events at HERA, J. Phys. G 39, 085009 (2012).

[34] F. A. Ceccopieri and L. Trentadue, A new fracture function approach to QCD initial state radiation, Phys. Lett. B 655, 15 (2007).

[35] G. Camici, M. Grazzini, and L. Trentadue, Fracture functions and jet calculus, Phys. Lett. B 439, 382 (1998).

[36] A. Vogt, S. Moch, and J. A. M. Vermaseren, The three-loop splitting functions in QCD: The singlet case, Nucl. Phys. B691, 129 (2004).

[37] S. Moch, J. A. M. Vermaseren, and A. Vogt, The three loop splitting functions in QCD: The nonsinglet case, Nucl. Phys. B688, 101 (2004).

[38] S. Alekhin, J. Blümlein, S. Moch, and R. Placakyte, Parton distribution functions, $\alpha_{s}$, and heavy-quark masses for LHC Run II, Phys. Rev. D 96, 014011 (2017).

[39] A. D. Martin, W. J. Stirling, R. S. Thorne, and G. Watt, Parton distributions for the LHC, Eur. Phys. J. C 63, 189 (2009).

[40] V. Bertone, A. Glazov, A. Mitov, A. Papanastasiou, and M. Ubiali, Heavy-flavor parton distributions without heavyflavor matching prescriptions, J. High Energy Phys. 04 (2018) 046. 
[41] R.S. Thorne, Effect of changes of variable flavor number scheme on parton distribution functions and predicted cross sections, Phys. Rev. D 86, 074017 (2012).

[42] R. D. Ball, V. Bertone, L. Del Debbio, S. Forte, A. Guffanti, J. Rojo, and M. Ubiali (NNPDF Collaboration), Theoretical issues in PDF determination and associated uncertainties, Phys. Lett. B 723, 330 (2013).

[43] R.S. Thorne, The effect on PDFs and $\alpha_{S}\left(M_{Z}^{2}\right)$ due to changes in flavour scheme and higher twist contributions, Eur. Phys. J. C 74, 2958 (2014).

[44] M. A. G. Aivazis, F. I. Olness, and W. K. Tung, Leptoproduction of heavy quarks. 1. General formalism and kinematics of charged current and neutral current production processes, Phys. Rev. D 50, 3085 (1994).

[45] R. S. Thorne and R. G. Roberts, An Ordered analysis of heavy flavor production in deep inelastic scattering, Phys. Rev. D 57, 6871 (1998).

[46] S. Forte, E. Laenen, P. Nason, and J. Rojo, Heavy quarks in deep-inelastic scattering, Nucl. Phys. B834, 116 (2010).

[47] R. S. Thorne, A Variable-flavor number scheme for NNLO, Phys. Rev. D 73, 054019 (2006).

[48] V. Bertone, S. Carrazza, and J. Rojo, APFEL: A PDF evolution library with QED corrections, Comput. Phys. Commun. 185, 1647 (2014).

[49] V. Bertone, S. Carrazza, N. P. Hartland, E. R. Nocera, and J. Rojo (NNPDF Collaboration), A determination of the fragmentation functions of pions, kaons, and protons with faithful uncertainties, Eur. Phys. J. C 77, 516 (2017).

[50] V. Bertone, N. P. Hartland, E. R. Nocera, J. Rojo, and L. Rottoli (NNPDF Collaboration), Charged hadron fragmentation functions from collider data, Eur. Phys. J. C 78, 651 (2018).

[51] M. Tanabashi et al. (Particle Data Group), Review of particle physics, Phys. Rev. D 98, 030001 (2018).

[52] R. D. Ball, S. Carrazza, L. Del Debbio, S. Forte, Z. Kassabov, J. Rojo, E. Slade, and M. Ubiali (NNPDF Collaboration), Precision determination of the strong coupling constant within a global PDF analysis, Eur. Phys. J. C 78, 408 (2018).

[53] S. Chekanov et al. (ZEUS Collaboration), Dissociation of virtual photons in events with a leading proton at HERA, Eur. Phys. J. C 38, 43 (2004).

[54] S. Chekanov et al. (ZEUS Collaboration), Deep inelastic scattering with leading protons or large rapidity gaps at HERA, Nucl. Phys. B816, 1 (2009).

[55] A. Aktas et al. (H1 Collaboration), Diffractive deep-inelastic scattering with a leading proton at HERA, Eur. Phys. J. C 48, 749 (2006).

[56] F. D. Aaron et al., Measurement of the cross section for diffractive deep-inelastic scattering with a leading proton at HERA, Eur. Phys. J. C 71, 1578 (2011).

[57] S. Chekanov et al. (ZEUS Collaboration), Measurement of dijet photoproduction for events with a leading neutron at HERA, Nucl. Phys. B827, 1 (2010).

[58] S. Chekanov et al. (ZEUS Collaboration), Dijet production in diffractive deep inelastic scattering at HERA, Eur. Phys. J. C 52, 813 (2007).

[59] F. D. Aaron et al. (H1 Collaboration), Measurement of dijet production in diffractive deep-inelastic scattering with a leading proton at HERA, Eur. Phys. J. C 72, 1970 (2012).
[60] S. Chekanov et al. (ZEUS Collaboration), Diffractive photoproduction of dijets in ep collisions at HERA, Eur. Phys. J. C 55, 177 (2008).

[61] F. D. Aaron et al. (H1 Collaboration), Diffractive dijet photoproduction in ep collisions at HERA, Eur. Phys. J. C 70, 15 (2010).

[62] V. Andreev et al. (H1 Collaboration), Measurement of dijet production in diffractive deep-inelastic ep scattering at HERA, J. High Energy Phys. 03 (2015) 092.

[63] V. Andreev et al. (H1 Collaboration), Diffractive dijet production with a leading proton in $e p$ collisions at HERA, J. High Energy Phys. 05 (2015) 056.

[64] C. Schmidt, J. Pumplin, C. P. Yuan, and P. Yuan, Updating and optimizing error PDFs in the Hessian approach, Phys. Rev. D 98, 094005 (2018).

[65] R. A. Khalek, S. Bailey, J. Gao, L. Harland-Lang, and J. Rojo, Towards ultimate parton distributions at the highluminosity LHC, arXiv:1810.03639.

[66] C. Willis, R. Brock, D. Hayden, T. J. Hou, J. Isaacson, C. Schmidt, and C. P. Yuan, Reduction of the PDF uncertainty in the high-mass Drell-Yan spectrum with ePump, arXiv: 1809.09481.

[67] P. Azzi et al. (HL-LHC Collaboration and HE-LHC Working Group), Standard Model Physics at the HLLHC and HE-LHC, arXiv:1902.04070.

[68] X. Cid Vidal et al., Beyond the Standard Model Physics at the HL-LHC and HE-LHC, arXiv:1812.07831.

[69] W. T. Giele and S. Keller, Implications of hadron collider observables on parton distribution function uncertainties, Phys. Rev. D 58, 094023 (1998).

[70] W. T. Giele, S. A. Keller, and D. A. Kosower, Parton distribution function uncertainties, arXiv:hep-ph/0104052.

[71] J. Pumplin, D. Stump, R. Brock, D. Casey, J. Huston, J. Kalk, H. L. Lai, and W. K. Tung, Uncertainties of predictions from parton distribution functions. 2. The Hessian method, Phys. Rev. D 65, 014013 (2001).

[72] R. D. Ball et al. (NNPDF Collaboration), Parton distributions from high-precision collider data, Eur. Phys. J. C 77, 663 (2017).

[73] R. D. Ball et al., Parton distributions with LHC data, Nucl. Phys. B867, 244 (2013).

[74] R. D. Ball, V. Bertone, M. Bonvini, S. Carrazza, S. Forte, A. Guffanti, N. P. Hartland, J. Rojo, and L. Rottoli (NNPDF Collaboration), A determination of the charm content of the proton, Eur. Phys. J. C 76, 647 (2016).

[75] H. Khanpour and S. Atashbar Tehrani, Global analysis of nuclear parton distribution functions and their uncertainties at next-to-next-to-leading order, Phys. Rev. D 93, 014026 (2016).

[76] T. J. Hou et al., Reconstruction of Monte Carlo replicas from Hessian parton distributions, J. High Energy Phys. 03 (2017) 099.

[77] A. D. Martin, R. G. Roberts, W. J. Stirling, and R. S. Thorne, Uncertainties of predictions from parton distributions. 1: Experimental errors, Eur. Phys. J. C 28, 455 (2003).

[78] S. Alekhin et al., HERAFitter, Eur. Phys. J. C 75, 304 (2015).

[79] D. Stump, J. Pumplin, R. Brock, D. Casey, J. Huston, J. Kalk, H. L. Lai, and W. K. Tung, Uncertainties of 
predictions from parton distribution functions. 1. The Lagrange multiplier method, Phys. Rev. D 65, 014012 (2001).

[80] J. Blumlein, H. Bottcher, and A. Guffanti, Non-singlet QCD analysis of deep inelastic world data at $O\left(\alpha_{s}^{3}\right)$, Nucl. Phys. B774, 182 (2007).

[81] F. James and M. Roos, Minuit: A system for function ginimization and analysis of the parameter errors and correlations, Comput. Phys. Commun. 10, 343 (1975); F. James, MINUIT function minimization and error analysis: Reference manual version 94.1, Report No. CERN-D-506, CERN-D506.

[82] M. Gluck, E. Reya, and A. Vogt, Parton distributions for high-energy collisions, Z. Phys. C 53, 127 (1992).
[83] F. D. Aaron et al. (H1 Collaboration), Measurement of leading neutron production in deep-inelastic scattering at HERA, Eur. Phys. J. C 68, 381 (2010).

[84] S. Chekanov et al. (ZEUS Collaboration), Leading neutron production in e $+\mathrm{p}$ collisions at HERA, Nucl. Phys. B637, 3 (2002).

[85] S. Chekanov et al. (ZEUS Collaboration), Leading proton production in deep inelastic scattering at HERA, J. High Energy Phys. 06 (2009) 074.

[86] A. Buckley, J. Ferrando, S. Lloyd, K. Nordström, B. Page, M. Rüfenacht, M. Schönherr, and G. Watt, LHAPDF6: Parton density access in the LHC precision era, Eur. Phys. J. C 75, 132 (2015). 